\title{
Plasmonic molecular assays: Recent advances and applications for mobile health
}

\author{
Tao Yu and Qingshan Wei $(\bowtie)$ \\ Department of Chemical and Biomolecular Engineering, North Carolina State University, 911 Partners Way, Campus Box 7905, \\ Raleigh, NC 27695, USA
}

\author{
Received: 16 March 2018 \\ Revised: 8 May 2018 \\ Accepted: 9 May 2018 \\ (C) Tsinghua University Press \\ and Springer-Verlag $\mathrm{GmbH}$ \\ Germany, part of Springer \\ Nature 2018

\section{KEYWORDS} \\ plasmonics, \\ molecular assay, \\ point-of-care diagnostics, \\ mobile health, \\ imaging, \\ sensing
}

\begin{abstract}
Plasmonics-based biosensing assays have been extensively employed for biomedical applications. Significant advancements in use of plasmonic assays for the construction of point-of-care (POC) diagnostic methods have been made to provide effective and urgent health care of patients, especially in resourcelimited settings. This rapidly progressive research area, centered on the unique surface plasmon resonance (SPR) properties of metallic nanostructures with exceptional absorption and scattering abilities, has greatly facilitated the development of cost-effective, sensitive, and rapid strategies for disease diagnostics and improving patient healthcare in both developed and developing worlds. This review highlights the recent advances and applications of plasmonic technologies for highly sensitive protein and nucleic acid biomarker detection. In particular, we focus on the implementation and penetration of various plasmonic technologies in conventional molecular diagnostic assays, and discuss how such modification has resulted in simpler, faster, and more sensitive alternatives that are suited for point-of-use. Finally, integration of plasmonic molecular assays with various portable POC platforms for mobile health applications are highlighted.
\end{abstract}

\section{Introduction}

Strategies that can offer accessible and affordable diagnosis of diseases with high selectivity and sensitivity are highly desired in the past decades. Generally, clinical diagnostics requires well-trained personnel to perform complicated assay protocols including sample collection, reaction, and analysis, all in centralized and well-equipped clinical settings, which is often costly and time consuming. However, in developing countries or poorly resourced regions, access to timely and adequate medical assistance may not even be available [1]. To address these issues, numerous research efforts have been devoted to the development of cost-effective and more accessible in vitro diagnostic (IVD) methods such as point-of-care tests (POCT). The global IVD market was estimated to be $\$ 74.1$ billion in 2017 and expected to reach $\$ 102.4$ billion by

Address correspondence to qwei3@ncsu.edu 
2022 at an estimated annual growth rate of $6.7 \%$ [2]. One of the main driving forces for the development of POC technologies is the increasing awareness of selfdiagnostics as a complement to doctoral examination [3]. As more and more diagnostic biomarkers being discovered, personalized or patient-side detection of diseases from easily accessible samples such as human blood becomes possible [4]. Common types of analytical biomarkers include proteins, nucleic acids, microbes, pathogens, and cells that can be obtained from various human specimens, such as blood, saliva, urine, and other bodily fluids [5]. The ultimate goal for all POC technologies under development is to provide a rapid "sample-to-answer" solution for identification of targeted diseases that requires little or no prepreparation steps and human intervention [6].

With the advances in wireless communication technologies, such as tablet computers, wearable devices, and mobile phones (especially smartphones), the concept of diagnosis and prognosis of diseases by a remote doctor is emerging $[7,8]$. Integrated with POC molecular assays, these consumer digital devicesenabled remote diagnostic platforms would significantly simplify the detection procedure, reduce the cost of detection systems, and improve the accessibility of medical diagnostics to poorly resourced individuals to participate in the health care matrix with both convenience and fidelity. This newly emerging area is frequently referred to "telemedicine", or "mobile health" in more general [9]. Although there is no convergent definition for "mobile health", it generally refers to all sorts of emerging POC technologies that can monitor, track, and transmit health metrics continuously and in real time. Fundamentally, mobile health is intended for analysis of samples derived from the human body to gather information for diagnostic and treatment purpose, and eventually offer a better healthcare for the patient [10].

Plasmonics, or surface plasmon resonance (SPR), has been an active research area of nanomaterials for decades. Plasmonics is a unique optical property of metallic nanostructures generated when their dimensions are smaller or comparable to the wavelength of incident light. Because of its tunability and large electromagnetic (EM) enhancement effect, it has attracted extensive attention for its promising applications in biosensing [11-15] and more recently in POC diagnostics $[16,17]$. This review examines the recent advances and applications of plasmonic technologies in POC diagnostics especially for protein and nucleic acid biomarker detection. It is however not intended to provide a comprehensive overview of various detection and sensing schemes that plasmonic nanomaterials can provide. For that, interested readers are referred to recent review articles elsewhere [11-15]. Rather, we particularly emphasize the past and on-going research efforts which try to integrate nanoplasmonics with conventional molecular assay methods such as protein immunoassays, nucleic acid amplification tests (NAAT), etc. to generate a set of plasmonics-modified assays that are simpler, more sensitive, and more accessible for translational mobile health applications. We organize the review with the following sections: In Section 2, we will introduce the basic principles of plasmonics and representative plasmonic technologies that have been used for molecular sensing. In Section 3, we will focus on how plasmonic technologies have been penetrating into conventional, "gold standard" molecular diagnostic methods toward highly sensitive detection of protein and nucleic acid targets. In Section 4 and 5, we will discuss the integration of plasmonic technologies with emerging portable assay and reader platforms, respectively, representing two important steps to transform current developments into POC tests. Finally in Section 6, the current limitations and challenges of using plasmonic molecular assays for POC applications will be highlighted and discussed.

\section{Principles of plasmonics and basic plasmonic technologies for molecular sensing}

SPR arises from the collective oscillation of conduction electrons at the interface of metal and dielectric materials under the resonant excitation of incident EM radiation (usually light). Generally, SPR can be divided into two categories: propagating surface plasmon polaritons (SPPs) and localized SPR (LSPR) [13]. SPPs rely on the propagating charge oscillations on a planar thin metal film when excited by light at a 
specific angle by using prism or grating couplers. The EM field of SPPs exponentially decreases away from the metal surface with a penetration depth of $\sim 200 \mathrm{~nm}$ into the dielectric layer. The SPP signal (resonant frequency and angle) can be easily modulated by the change of refractive index of the surrounding medium, making it an ideal label-free refractive index sensor for monitoring surface binding events. Different from SPPs, LSPR is a confined oscillation of conduction electrons at the surface of metallic nanostructures such as nanoparticles, nanowires, or nanoholes that are similar or smaller in size than the wavelength of incoming light. LSPR is a near-field phenomenon, where the EM field of LSPR is highly concentrated around the nanostructures with a much shorter decay length (typically $10 \mathrm{~nm}$ ) than SPPs $[14,18]$.
Plasmonic materials are usually made of noble metals (e.g., gold, silver, or alloys) $[19,20]$. With the advance of the nanoengineering technology, the characteristic SPR properties of nanomaterials can be fully adjusted by tuning the size, shape, and composition of plasmonic materials, as well as the dielectric properties of surrounding environments [21, 22]. Strategies for functionalization of the surface of these materials have also been well studied, bringing in molecular specificity to plasmonic substrates for selective binding and detection of target molecules of interest for chemical and biological sensing [23, 24]. Various plasmonic sensing mechanisms have been demonstrated in recent years, and the basic plasmonicsenabled sensing technologies are summarized below (also see Table 1).

Table 1 Representative examples of various plasmonic technologies used for molecular detection ${ }^{\mathrm{a}}$

\begin{tabular}{|c|c|c|c|c|c|c|}
\hline Biomarker & Disease & Materials & Binding layer & Approach & LOD & Ref. \\
\hline Infliximab & IBD & $\begin{array}{c}\text { Fiber-optic } \\
\text { with Au surface }\end{array}$ & antibody & SPR & $2 \mathrm{ng} / \mathrm{mL}$ & {$[46]$} \\
\hline Tau & $\mathrm{AD}$ & $\mathrm{Au}$ film & $\begin{array}{l}\text { Antibody/ } \\
\text { aptamer }\end{array}$ & SPR & $10 \mathrm{fM}$ & {$[47]$} \\
\hline $\begin{array}{l}\text { A } \beta 1-40 \\
\text { A } \beta \text { 1-42; } \\
\tau \text { protein }\end{array}$ & $\mathrm{AD}$ & $\mathrm{Au}$ NPs & Antibody & LSPR & $\begin{array}{l}34.9 \mathrm{fM} ; \\
26 \mathrm{fM} \\
23.6 \mathrm{fM}\end{array}$ & [48] \\
\hline $\begin{array}{c}\text { Salmonella, Listeria, } \\
\text { E. coli } \mathrm{O} 157\end{array}$ & Pathogen & $\mathrm{Au}$ NPs & Antibody & Plasmon coupling & $6.7 \mathrm{aM}$ & [49] \\
\hline DNA & HIV & $\mathrm{Au}$ NPs & DNA & Plasmon coupling & 0.01 zeptomoles & {$[50]$} \\
\hline Enterovirus 71 & Virus infection & $\mathrm{Au}$ NPs & Antibody & Plasmon coupling & $0.65 \mathrm{ng} / \mathrm{mL}$ & {$[51]$} \\
\hline $\begin{array}{c}\text { MERS-CoV; } \\
\text { MTB; } \\
\text { HPV }\end{array}$ & Infectious diseases & Ag NPs & PNA & Plasmon coupling & $\begin{array}{l}1.53 \mathrm{nM} ; \\
1.27 \mathrm{nM} ; \\
1.03 \mathrm{nM}\end{array}$ & {$[52]$} \\
\hline Thrombin & - & $\mathrm{Au}$ NPs and $\mathrm{Au}$ film & DNA & SERS & $86 \mathrm{pM}$ & {$[53]$} \\
\hline $\begin{array}{c}\text { AFP } \\
\text { GPC-3 }\end{array}$ & Liver cancer & Ag NP films & Antibody & SERS & $0.1 \mathrm{pM}$ & {$[54]$} \\
\hline DNA & HIV-1 & $\mathrm{Au}$ NPs & DNA & SERS & $0.24 \mathrm{pg} / \mathrm{mL}$ & {$[55]$} \\
\hline DNA & & Au NR & DNA & SEF & $10 \mathrm{pM}$ & [56] \\
\hline antibodies & SLE & Au film & Peptide & SEF & $10 \mathrm{pg} / \mathrm{mL}$ & {$[57]$} \\
\hline $\begin{array}{l}\text { CEA; } \\
\text { Cyfra21-1; } \\
\text { NSE }\end{array}$ & Lung cancer & Au nanoislands & Antibody & SEF & $\begin{array}{l}0.19 \mathrm{ng} / \mathrm{mL} \\
0.05 \mathrm{ng} / \mathrm{mL} \\
0.15 \mathrm{ng} / \mathrm{mL}\end{array}$ & {$[58]$} \\
\hline PSA & - & Au nanoarray & Antibody & SEF & $100 \mathrm{pg} / \mathrm{mL}$ & {$[59]$} \\
\hline
\end{tabular}

${ }^{a}$ Limit of detection (LOD), amyloid beta 1-40 (A $\beta$ 1-40), amyloid beta 1-42 (A $\beta$ 1-40), tau protein ( $\tau$ protein), Alzheimer's disease (AD), inflammatory bowel disease (IBD), $\alpha$-fetoprotein (AFP), glypican-3 (GPC-3), carcinoembryonic antigen (CEA), neuron-specific enolase (NSE), systemic lupus erythematosus (SLE), peptide nucleic acid (PNA), middle east respiratory syndrome coronavirus (MERS-CoV), Mycobacterium tuberculosis (MTB), prostate specific antigen (PSA), and human papillomavirus (HPV). 


\subsection{SPR sensor}

SPR sensing is a sensitive technique that relies on the measurement of refractive index changes which occur in the evanescent field of metal-dielectric interfaces to detect the presence of target molecules [25-28]. In a typical setup (Fig. 1(a)), a thin metal film is used as SPR substrate and molecular recognition ligands (e.g., aptamer or antibodies) are immobilized on the surface of the SPR sensor; light excitation is delivered to the sensor substrate from the backside of the substrate through a prism to generate SPP. Once a target analyte is captured on the substrate and the local refractive index is altered, the SPR sensor detects the binding event by monitoring the intensity, wavelength, or angular spectral shifts of the reflected or transmitted SPR resonance spectra. Therefore, SPR sensing is a completely label-free technique for probing biomolecular interactions without the need of additional tags or labels [29]. SPR sensing has been extensively used for characterization and quantification of a series of proteins, DNA, pathogens, and many other biomarkers.
SPR sensing can also be enabled by LSPR generated from individual plasmonic nanostructures such as metallic nanoparticles, nanorods, and nanoprisms, or periodic plasmonic nanoarrays [33,34]. The detection principle of LSPR sensors is quite similar to those of thin-film based SPR sensors as discussed above, but LSPR sensors have their own advantages. In LSPR sensors, the resonant spectra can be generated by direct coupling of light to the sample without the need of a prism or grating coupler; therefore, the incidence angle does not need to be precisely controlled. In addition, LSPR can be excited by using simple broadband white light. As a result, LSPR sensing can be performed with low-cost and compact light sources such as light emission diodes (LED). The refractive index sensitivity of LSPR, defined as the shifted wavelength per refractive index unit, or $\mathrm{nm} / \mathrm{RIU}$, is highly tunable and dependent on the size, shape, and composition of the nanomaterials being used. In general, plasmonic nanostructures containing sharp features exhibit higher index sensitivity. For example, gold nanostars exhibit a large index sensitivity of $703 \mathrm{~nm} / \mathrm{RIU}$ while gold nanosphere's index sensitivity is as small as (a)

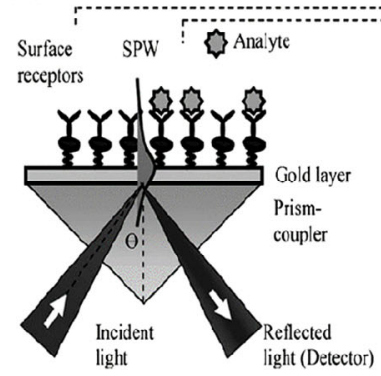

(c)

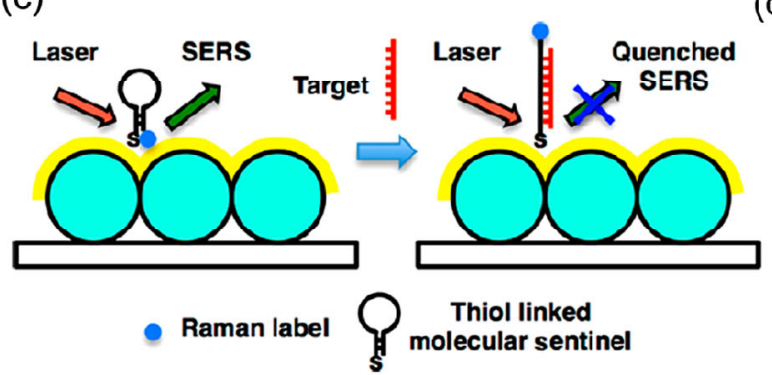

(b)

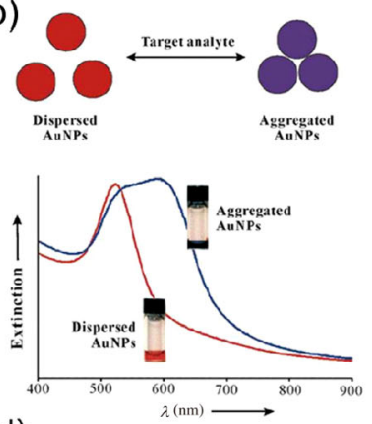

(d)

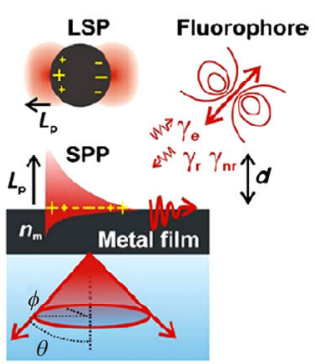

Figure 1 Schematic illustration of various plasmonic sensing approaches. (a) Prism-coupled label-free SPR sensor: binding event (left) and the corresponding resonance shift (right) (reproduced with permission from Ref. [27], (C) Elsevier 2007). (b) Schematic representation of colorimetric $\mathrm{Au} \mathrm{NP}$ aggregation assay based on plasmonic coupling (reproduced with permission from Ref. [30], C Wiley-VCH 2008). (c) SERS-based DNA detection via probe hybridization (reproduced with permission from Ref. [31], C American Chemical Society 2013). (d) Schematic of SEF: interaction between the dipole of a fluorophore and the confined field of SPP or LSPR (reproduced with permission from Ref. [32], (C) Springer Nature 2014). 
$44 \mathrm{~nm} / \mathrm{RIU}$ [35]. Because of much simpler instrumentation requirement of LSPR compared to conventional SPR technology and the high detection sensitivity, LSPR has demonstrated great translational potential for POC applications. Indeed, various POC LSPR sensing applications have been demonstrated in the last decade, ranging from clinical diagnostics to environmental sensing [36, 37].

\subsection{Plasmon coupling sensor}

Resonant spectral shift can be modulated not only by refractive index change but also by plasmon coupling effect. Plasmon coupling occurs when two plasmonic nanoparticles approach to each other to a distance of a few nanometers, where the resonance of individual particles starts to hybridize, resulting in a red-shift of the spectra (Fig. 1(b)). Such changes turn the plasmonic nanoparticles into a colorimetric assay, in which the degree of color change (spectral shift) can be correlated to the concentration of analytes (the trigger molecules of nanoparticle aggregation). Plasmonic colorimetry is one of the most competitive biosensing technologies available for POC testing due to its simplicity, sensitivity, and reliability [38]. For example, Mirkin et al. have pioneered on this format of assay using oligonucleotidefunctioned gold nanoparticles (Au NPs), which exhibit a strong red shift upon aggregation in the presence of target nucleotide $[39,40]$, allowing rapid and sensitive readout with the naked eye or by an inexpensive optical device [41]. In addition to aggregation of NPs, colorimetric plasmon detection could also be demonstrated by growth or etching of NPs to generate colorimetric signal changes [22, 42, 43].

\subsection{Surface-enhanced Raman scattering (SERS)}

SERS is a surface spectroscopic technology providing biosensor applications with rich molecular structure information (Fig. 1(c)). SERS is also a plasmonics-based technique which relies on nanostructured substrates to generate concentrated EM field through the formation of plasmonic hot spots to amplify the intrinsically weak Raman scattering process [44]. SERS spectroscopy measures the molecular vibrations to provide fingerprint signatures of target molecules [45], and therefore is less susceptible to the interference of measurement environment compared to the SPR technology. Both excitation and scattering wavelength of SERS can be tailored into the near-infrared biological transparency window, which greatly suppresses the background of complex biological samples and thus improves the detection signal-to-noise ratios (SNR). In SERS, the spontaneous Raman scattering cross section can be enhanced up to 10 orders of magnitude when the Raman tags are adsorbed on rough metal surfaces, attributed to the combination of an EM mechanism due to SPR and a chemical mechanism related to charge transfer between the substrate and the SERS molecules. As a result, SERS spectroscopic measurement can achieve ultrahigh detection sensitivity down to the single-molecule level by using densely packed metal nanostructures (e.g., $\mathrm{Au}, \mathrm{Ag}$, and $\mathrm{Cu}$ ) on a glass substrate $[60,61]$.

Various SERS substrates have been demonstrated. Early SERS assays were performed on roughened silver electrodes, where single-molecule sensitivity was demonstrated [62-65]. Silver colloids are the most widely used SERS substrates due to the large extinction cross section of silver in the visible spectral region. The spectral range of silver nanostructures can be flexibly adjusted by adjusting particle size and shape. Recently, single-nanoparticle SERS tags are also emerging, allowing SERS measurement to be performed in vivo [66]. With the recent advance of portable Raman spectrometers and commercially available SERS substrates, this ultrasensitive and noninvasive method has shown great promise for molecular fingerprinting in the field, which is extremely useful for forensic science and a broad range of POC applications [67, 68].

\subsection{Surface-enhanced fluorescence (SEF)}

Metal-enhanced fluorescence (MEF), or surfaceenhanced fluorescence (SEF), offers an attractive alternative for amplification of fluorescent signals in bioassays through the coupling of fluorescence with surface plasmons (Fig. 1(d)). When a fluorophore is positioned in a close proximity to a metal film or nanoparticle, both excitation rate and radiative decay rate (rate of fluorescence emission) of the fluorophore are enhanced as a result of enhanced EM field near the metal surface. The enhancement factor (EF) of fluorescence signals varies from around 10-fold for easy-to-fabricate planar metal thin films [69-71] to 
more than 1,000-fold for precisely engineered nanoantennas [72-74]. Roughened gold-island films or deposited gold nanoparticle monolayers have shown a good balance between the simplicity of substrate preparation and relatively high EF factors (10-100 folds). Combined with protein microarray technology, these substrates have recently been explored for enhancing protein detection in fluorescent immunoassays [75-78].

\section{Emerging plasmonic molecular assays for biodetection}

Molecular diagnostics relies on specific detection of biomarkers which could be extremely low in abundance. It then becomes a big challenge for detection by using traditional means. Thus, there is an immediate need for the development of ultrasensitive assay methods capable of trace-level biomarker detection. In this regard, plasmonic biosensors have shown a great promise. Plasmonic technology has been engaged in molecular detection in various different formats: from enhancing end-point signal detection, serving as alternative optical labels, to recently being fully involved in the assay process to accelerate, simplify, and amplify biochemical reactions. This potentially opens a new branch of molecular assay technology and POC diagnostics. Here, based on the types of sensing targets (proteins or nucleic acids), various plasmonic molecular assays are summarized and introduced.

\subsection{Plasmonic assays for protein detection}

Proteins are large biomolecules, playing an important role in living organisms, including catalyzing metabolic reactions, replicating DNA, responding to stimuli, and molecule transportation. Protein detection mainly relies on specific molecular binding, including immunological recognition (antibody-antigen interaction), aptamerprotein interaction, and peptide-protein interaction. Plasmonics has been extensively applied in protein biomarker detection to improve detection sensitivity and specificity in diagnosis and monitoring of a variety of diseases [18, 79]. We focus on reviewing the implementation of plasmonics in antibody-based recognition as an example. The same optical enhan- cement methodology in principle can also be applied to other protein assays based on aptamers and peptides.

\subsubsection{Plasmonic enzyme-linked immunosorbent assay ( $p E L I S A)$}

Enzyme-linked immunosorbent assay (ELISA) is the most common method used in laboratories for protein detection and identification since 1971 [80]. In a conventional sandwich ELISA, the analyte (e.g., antigen) is firstly captured by the capturing antibody immobilized on the substrate, and then targeted with a detection antibody to form a sandwich structure (Fig. 2(a)). Afterwards, an enzyme-conjugated secondary antibody is introduced and binds to the primary detection antibody for signal amplification. The biocatalytic reactions then generate colorimetric or fluorescent signals that can be quantified by a plate reader or spectrophotometer. The colorimetric/ fluorometric signal is proportional to the number of enzymes attached to the immunocomplex and therefore the number of antigens captured.

Colorimetric ELISA doesn't require complex measurement instrument using special light sources and filters, and the results can be easily visible with the naked eye. Therefore, colorimetric ELISA is attractive for POC use where expensive and bulky plate readers are not available [81]. However, one of the main limitations for conventional colorimetric ELISA is its relative low detection sensitivity compared with other detection approaches, such as fluorescent assays or SERS [82]. When low concentrations of analytes are present, the color change of solutions will likely be too subtle to be distinguished from the noncolored controls [83].

A potential solution is to use plasmonic metallic nanoparticles instead of organic fluorophores as reporter molecules, since metallic nanoparticles have orders of magnitude larger absorption cross section than organic dyes. Based on this principle, Stevens et al. have developed a plasmonic ELISA (pELISA) utilizing analyte-dependent $\mathrm{Au}$ NP growth and therefore the different color of Au NPs as the reporting signals of the enzymatic assay (Fig. 2(b)) [83-85]. As shown in Fig. 2(b), pELISA differs from conventional colorimetric ELISA in the choice of enzyme molecules. In pELISA, a catalase was conjugated to the secondary 
(a)

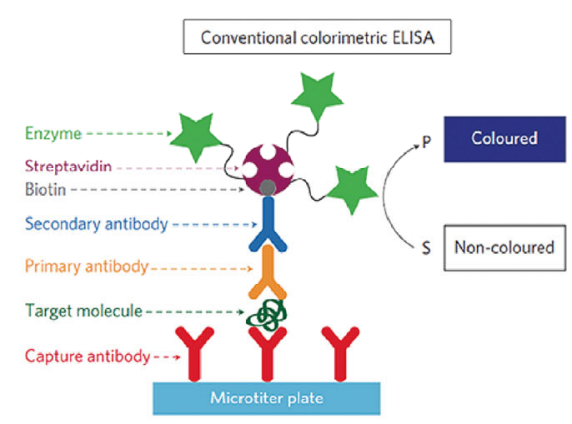

(b)

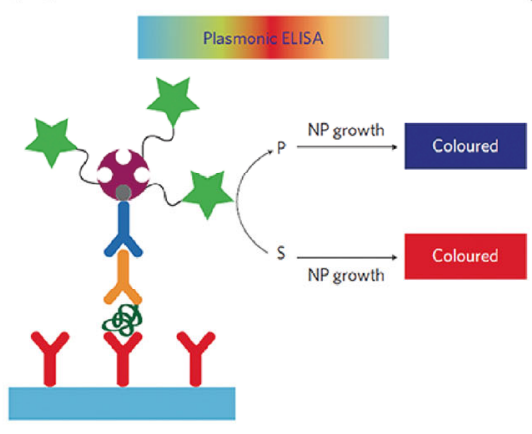

(c)

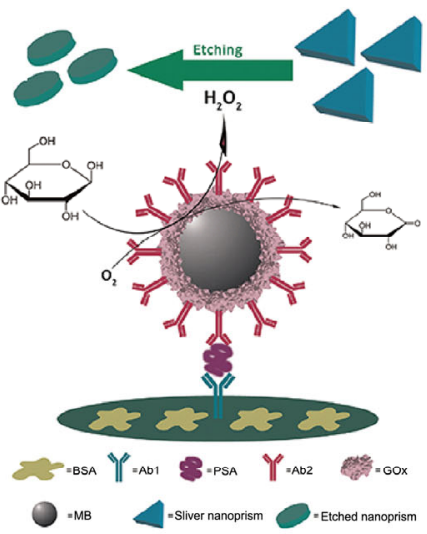

(d)

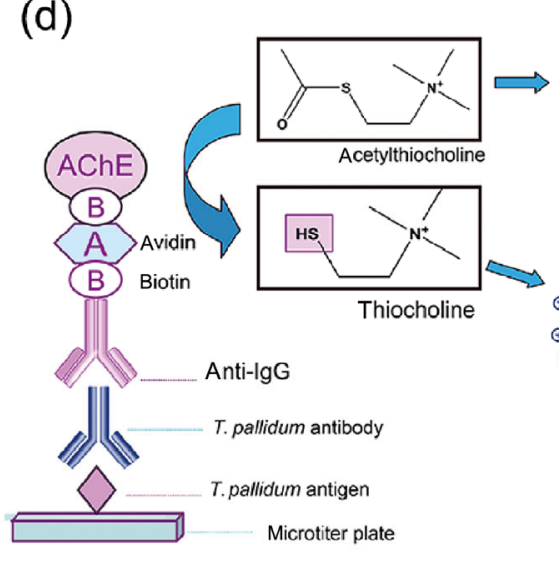

Q 230

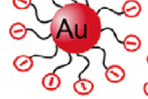

नी Au

Citrate-Capped AuNPs,

non-aggregated

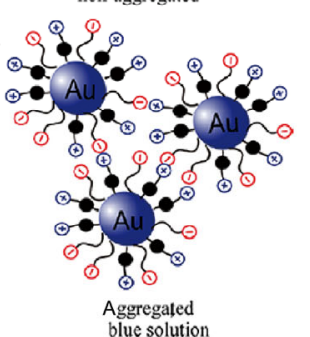

(e)

Figure 2 Principles of different pELISA. (a) Conventional colorimetric ELISA. (b) pELISA based on nanoparticle growth (reproduced with permission from Ref. [83], (C) Springer Nature 2012). (c) pELISA based on nanoparticle reshaping (reproduced with permission from Ref. [92], (C) Elsevier 2015). (d) pELISA based on nanoparticle aggregation (reproduced with permission from Ref. [93], (C) Elsevier 2014). (e) Single-molecule pELISA (reproduced with permission from Ref. [96], (C) American Chemical Society 2011).

antibody which consumes hydrogen peroxide $\left(\mathrm{H}_{2} \mathrm{O}_{2}\right)$, a reducing agent capable of converting gold precursor $\left(\mathrm{Au}^{3+}\right)$ into atomic gold to form $\mathrm{Au}$ NPs. Increasing analyte level leads to lower $\mathrm{H}_{2} \mathrm{O}_{2}$ concentration, therefore slower growth kinetics of $\mathrm{Au} \mathrm{NPs}$, and eventually aggregation of Au NPs due to poor control of growth rates. As a result, the assay turns into blue. In contrast, the color of the assay solution is more red with lower concentrations of analyte. In proof-ofconcept experiments, pELISA was applied for detecting prostate specific antigen (PSA) [83], HIV-1 capsid antigen p24 [83], and HIV-1 protein gp120 [85] in whole serum or FBS-spiked solutions with a detection limit in the range of $10^{-18}-10^{-17} \mathrm{~g} / \mathrm{mL}$, orders of magnitude lower than the LOD of conventional ELISA (pg-ng/mL range). Later on, Long et al. reported a plasmonic ELISA strategy for the detection of hepatitis B surface antigen (HBsAg) and $\alpha$-fetoprotein (AFP) using alcohol dehydrogenase $(\mathrm{ADH})$ catalyzed growth of Au NPs [86]. In this assay, the antibody-conjugated $\mathrm{ADH}$ catalyzed the reaction between NAD+ and ethanol to generate $\mathrm{NADH}$ and acetaldehyde, and NADH reduced $\mathrm{Au}^{3+}$ to $\mathrm{Au}$ NPs subsequently. The proposed method can visually detect protein biomarkers with the lowest concentration down to $1.0 \times 10^{-12} \mathrm{~g} / \mathrm{mL}$.

In general, the labeling density of enzyme molecules is limited to one enzyme per antibody. NPs with proper size on the other side can act as multiple binding site carriers to increase the loading density of enzymes, and therefore further amplifies the immunoassay signal. This strategy has been first demonstrated in conventional ELISA [87] and recently in pELISA. Chen et al. reported a quantitative pELISA immunoassay based on glucose oxidase (GOx)-catalyzed growth of 
$5 \mathrm{~nm}$ Au NPs [88]. The detection antibody was labeled with a magnetic bead (MB, $1 \mu \mathrm{m}$ in diameter), where each MB carried around 76,000 GOx molecules instead of only one enzyme per antibody in conventional ELISA. As a result, the LOD of PSA of this approach (93 aM) is more than 4 orders of magnitude lower than that of commercial ELISA (6.3 pM). In fact, the use of high surface-to-volume ratio of nanoparticles as enhancing substrates has been used as a general strategy to improve the loading densities of various other components of ELISA, such as capturing antibody [89], detection antibody, or both [90, 91].

In addition to nanoparticle growth, enzymatic strategy has also been applied to reshape or aggregate plasmonic nanoparticles in order to induce spectral shift for highly sensitive detection. Tang et al. developed a pELISA to detect PSA by etching silver nanoprisms from triangles into smaller spherical Ag NPs using GOx-generated $\mathrm{H}_{2} \mathrm{O}_{2}$ (Fig. 2(c)) [92]. The SPR peak shift enables the detection of PSA with a LOD of $4.1 \mathrm{fg} / \mathrm{mL}$, more than 5 orders of magnitude better than that of conventional HRP-based ELISA $(1.25 \mathrm{ng} / \mathrm{mL})$. Jiang et al. reported a pELISA strategy using enzymemediated aggregation of Au NPs for detection of T. pallidum antibodies [93]. As shown in Fig. 2(d), a serine protease, AChE, was conjugated to the secondary antibody, which hydrolyzes acetylthiocholine to produce thiocholine. The thiol product in turn attacks citratestabled Au NPs to induce NP aggregation. A LOD of $0.98 \mathrm{pg} / \mathrm{mL}$ was successfully achieved by this pELISA.

Single-molecule ELISA has been explored in the past years by employing the digital assay approach, where ELISA reaction was performed in microwells and each microwell monitors a single-molecule event governed by the Poisson distribution [94]. Alternatively, single-molecule ELISA can also be achieved on a plasmonic substrate. Kall et al. developed a pELISA technology by combing LSPR refractive index sensing and enzymatic reaction with its LOD approaching single-molecule sensitivity $[95,96]$. In their design, an array of Au NPs with conical or rod shapes were used as the LSPR substrate. Each plasmonic NP only binds one or a few HRP molecules that catalyzed a localized precipitation reaction at the particle surface, resulting a dramatically amplified plasmonic shift in the resonant scattering spectra (Fig. 2(e)). Although this method may suffer from inhomogeneous sensing properties of individual NPs, it opens up a brand new avenue towards single-molecule ELISA by harnessing the unique properties of nanoplasmonics.

\subsubsection{Nonenzymatic plasmonic immunoassays}

Different from ELISA that uses enzyme reactions to generate and amplify the output signals, nonenzymatic plasmonic immunoassay refers to sandwich immunoassays which use nanoparticles or fluorogenic reporters as labels to generate plasmonics-enhanced signals for assay quantification in the absence of enzymatic amplification. It greatly simplifies the assay protocols by eliminating the enzymatic reaction step.

Plasmonic NPs (e.g., Au or Ag NPs) have been frequently used as labeling tags in nonenzymatic protein immunoassays. The most common example is perhaps the lateral flow assay (LFA, see details in Section 4.2), where antibody-conjugated Au NPs are used as color reporters to bind antigen that has been captured by the immobilized antibody on the paper substrate. The signaling mechanism is based on the plasmon-resonant absorption and scattering of $\mathrm{Au}$ NPs to produce distinguishable colorimetric contrast. To improve the detection sensitivity, the assay can be followed by silver enhancement treatment where $\mathrm{Au}$ NPs serve as catalysts and nucleation centers for silver reduction and precipitation $[97,98]$. The resulted silver coating turns the assay spots into black, which is more easily detectable against the background noise.

$\mathrm{Au}$ and other metallic NPs could be excellent signal enhancement tags for SPR-based protein sensing. Conventional SPR sensing utilizes a monolayer of surface-immobilized capturing antibody to detect specific antigen and generates resonance spectral shift upon molecular binding. Due to the existing of the antibody layer, the index sensitivity of the SPR substrate to the antigen binding is reduced. A solution to circumvent this issue is to introduce a secondary antibody labeled with an amplifier Au NP tag to form a "film-analyte-particle" sandwich structure. The Au amplifiers interact with the planar Au substrate to generate plasmon coupling, which significantly enlarges the resonant shift (Fig. 3(a)) [99]. Natan et al. demonstrated that antibody-Au colloid conjugates could result in a 25-fold larger signal than that of free 
(a)

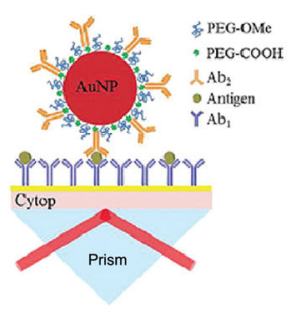

(c)

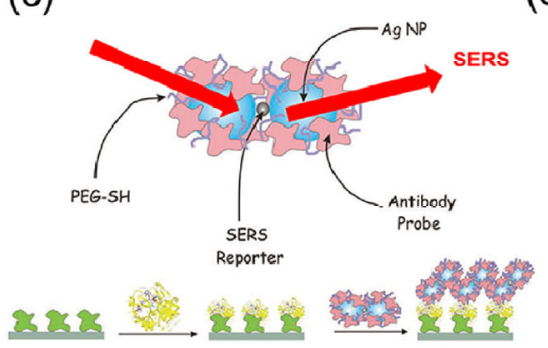

(b)

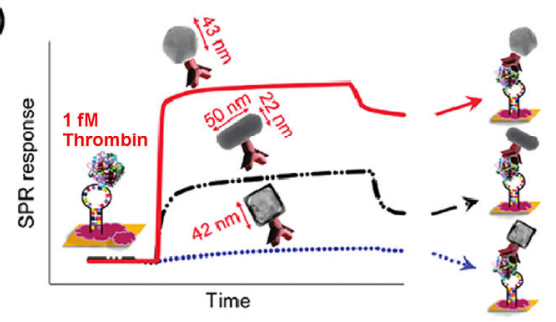

(d)

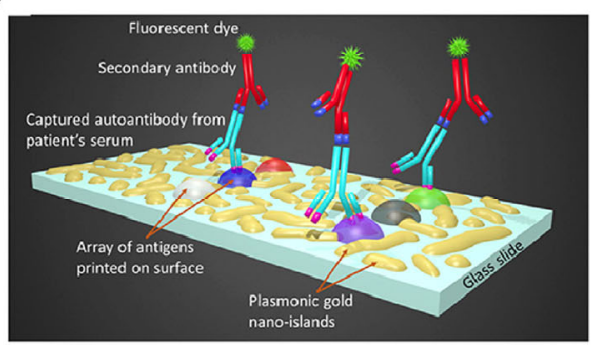

Figure 3 Plasmonics-enhanced nonenzymatic immunoassays. (a) NP-enhanced SPR sensing (reproduced with permission from Ref. [99], (C) Royal Society of Chemistry 2016). (b) Effect of NP shape and size on SPR signal enhancement (reproduced with permission from Ref. [101], (C) American Chemical Society 2012). (c) SERS-based NP tags for protein detection (reproduced with permission from Ref. [102], (C) Wiley-VCH 2010). (d) Plasmonic substrates for SEF detection of proteins combined with fluorescent tags (reproduced with permission from Ref. [103], (C) National Academy of Sciences 2017).

antibody that was 6 orders of magnitude more concentrated [100]. Lee et al. reported that nanoparticleenhanced SPR is highly dependent on the shape and size of amplifier nanoparticles [101]. As shown in Fig. 3(b), three shapes of Au NPs, including nanorods, nanocages, and quasi-spherical NPs were used in this study, and quasi-spherical NPs resulted in the greatest enhancement in sensitivity with a LOD of $1 \mathrm{aM}$ for thrombin. In comparison, nanorod- and nanocageantibody conjugates showed LODs of $10 \mathrm{aM}$ and $1 \mathrm{fM}$, respectively. These results represent 3 orders of magnitude improvement compared to the previous results of using smaller $(\sim 15 \mathrm{~nm}) \mathrm{Au}$ NPs due to a combination of improved surface passivation and proper selection of amplifier nanoparticles. Recently, Thierry et al. revisited the enhancement mechanism of the nanoparticle-film hybrid system and found that the long-range surface plasmon resonance (LRSPR) had been playing a significant role in enhancing resonance shift [99]. They demonstrated in both experimental measurement and theoretical model that 50 -fold improvement in sensitivity could be obtained when using $50 \mathrm{~nm} \mathrm{Au} \mathrm{NP} \mathrm{tags} \mathrm{in} \mathrm{comparison} \mathrm{to}$ tag-free detection of CEA [99]. In addition to SPR, introducing plasmonic NP tags to the sandwich immunoassays also opens up other detection possibility such as SERS. For example, Bazan et al. demonstrated a SERS-based detection tag consisting of an Ag NP dimer and a SERS reporter that are functionalized by probe antibodies (Fig. 3(c)) [102]. A LOD of $100 \mathrm{pM}$ was demonstrated for detection of human $\alpha$-thrombin.

Plasmonic nanostructures could also been used as optically active substrates for enhancing protein immunoassays via SEF. Compared with other tags, fluorogenic tags are one of the most widely used labeling agents for antibody detection because of its simplicity, high sensitivity, and capability for multiplexing. However, organic fluorophores suffer from variations in quantum yield and therefore detection efficiency. Plasmonic nanosubstrates are the ideal candidates to enhance the fluorescence signal intensity especially for low quantum yield dyes through SEF (see principle of SEF in Section 2.4). For example, Dai et al. developed a plasmonics-enhance fluorescence immunoassay using porous gold films with nearinfrared fluorescence enhancement (NIR-FE) of up to 100-fold for multiplexed protein detection with femtomolar sensitivity over a broad dynamic range (Fig. 3(d)) [76, 77, 103]. In this design, the capturing antibody or antigen was immobilized on a gold 
nanoisland film with abundant nanogaps $(\sim 10 \mathrm{~nm}$ range). After capturing of target analytes (antigen or antibody), a NIR fluorophore-conjugated secondary antibody was added to the substrate to generate SEF signal. The plasmonic slide was demonstrated to be compatible with standard microarray scanner for signal quantification. The system was first tested for detection of a model cancer biomarker, CEA, at concentrations down to $\sim 5 \mathrm{fM}$ [76], and later for diagnosis of type 1 diabetes (T1D) by detection of GAD65- or IA2-specific autoantibodies from $2 \mu \mathrm{L}$ of human blood [77]. Recently, the SEF nano-gold chip technology has also been explored for the early detection of hypertensive heart disease by multiplexed screening of a panel of 10 cardiovascular autoantibodies simultaneously [103].

\subsection{Plasmonic assays for nucleic acid detection}

Nucleic acids are essential for all known forms of life. Deoxyribonucleic acid (DNA) is a molecular that carries the genetic information and translates the genetic code into proteins, while ribonucleic acid (RNA) plays various biological roles in coding, decoding, regulation and expression of genes. Consequently, detection of nucleic acids provides considerable information for biological and medical diagnostics that other methods cannot provide [104].

\subsubsection{Plasmonics-enhanced polymerase chain reaction (PCR)}

Since its invention in 1983 by Kary Mullis, PCR has become the most widely used technique for clinical molecular diagnostics, and is still considered the "gold standard" for amplification and detection of lowabundance nucleic acids [105]. A single copy or a few copies of DNA segments can be amplified into thousands to millions of copies of DNA sequences within tens of minutes via the PCR process. However, thermal cycling of PCR remains a relatively time consuming and complex process, which makes PCR not available for rapid POC use in early days. In recent years, however, many efforts have been devoted to improving the performance of PCR by either miniaturizing thermocyclers [106], automating assay process with microfluidics [107], or developing costeffective and field-portable readout platforms [108-110].
These advancements have substantially improved the accessibility of PCR.

Plasmonic technologies have been engaged in PCR assays mainly in two different ways: 1) enhancing PCR product detection via SPR, SERS, SEF, or plasmonic coupling approaches, and 2) accelerating the rate of PCR assays through localized plasmonic heating. PCR products are conventionally detected by gel electrophoresis or fluorometry based on intercalating double-stranded DNA with fluorescent dyes. However, the former is not possible to quantitatively determine the concentration of PCR products, and the latter is limited by detection sensitivity and number of detection channels for multiplexing.

Alternatively, various plasmonics-based detection methods have been coupled to PCR assays to quantify PCR products more accurately and sensitively. Karube et al. demonstrated the feasibility of using SPR for sensitive detection of PCR products with picomolar sensitivity [111]. The method relies on a peptide nucleic acid (PNA) probe that was immobilized on a commercially available SPR sensor chip to capture the sense strands of denatured PCR products. Neutral PNA probes showed better hybridization efficiency with PCR products than DNA probes, partially because neutral PNA probes allowed the application of low-salt condition, in which both inter- and intra-molecular structure of PCR products were minimized for more efficient "probe-target" hybridization. With this SPR chip, a LOD of 7.5 pmol of the target DNA was demonstrated. Later, the same group reported a different strategy to bind PCR products onto the SPR substrate [112]. In this new approach, two asymmetric PCR reactions were performed in a single pot to amplify the sense and antisense strands of the target DNA, respectively, but with different lengths (Fig. 4). The two single-stranded products were then annealed to form double-stranded detection sequences with a toehold that were captured and detected by oligofunctionalized SPR sensor. Ding et al. developed an intensity-based SPR imaging (SPRi) system combining DNA microarray technology for quantification of denatured PCR products [113]. This system has been used for detection of the BCR/ABL fusion gene in chronic myelogenous leukemia (CML) with a LOD of $10.29 \mathrm{nM}$. 

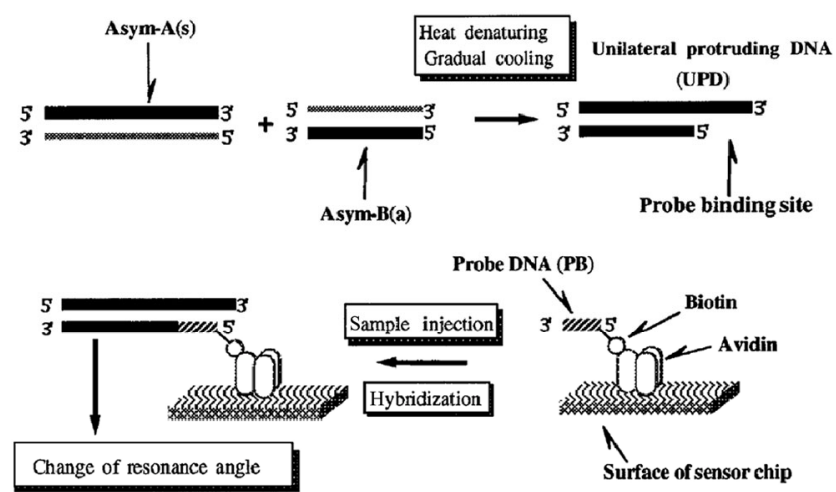

Figure 4 Detection of PCR products using SPR (reproduced with permission from Ref. [112], (C) American Chemical Society 1999).

PCR products can also be quantified by SEF or SERS. Knoll et al. demonstrated the detection of denatured PCR products with SEF by introducing fluorescently labeled primers in the amplification, and ssDNA probe-functionalized gold chip for fluorescence enhanced detecton [114]. This SEF platform allowed for real-time monitoring of hybridization processes with a LOD of $100 \mathrm{fmol}$ on the PNA probe surface and $500 \mathrm{fmol}$ on the DNA probe surface. Barnes et al. reported the detection of denatured PCR products by SERS with a commercially developed PCR-SERS assay system [105]. Not only being more sensitive to fluorescence detection mode [115], SERS is also capable of greatly expanding the throughput of PCR by multiplexed detection of up to 10 targets per reaction [105]. Graham et al. implemented a different SERS detection strategy for PCR [116]. They functionalized normal PCR primers with SERS probes, a hybrid NP containing both SERS tag molecules and Ag NPs as enhancement substrates (Fig. 5(a)). Using SERS primers, the PCR products were directly available for SERS quantification.
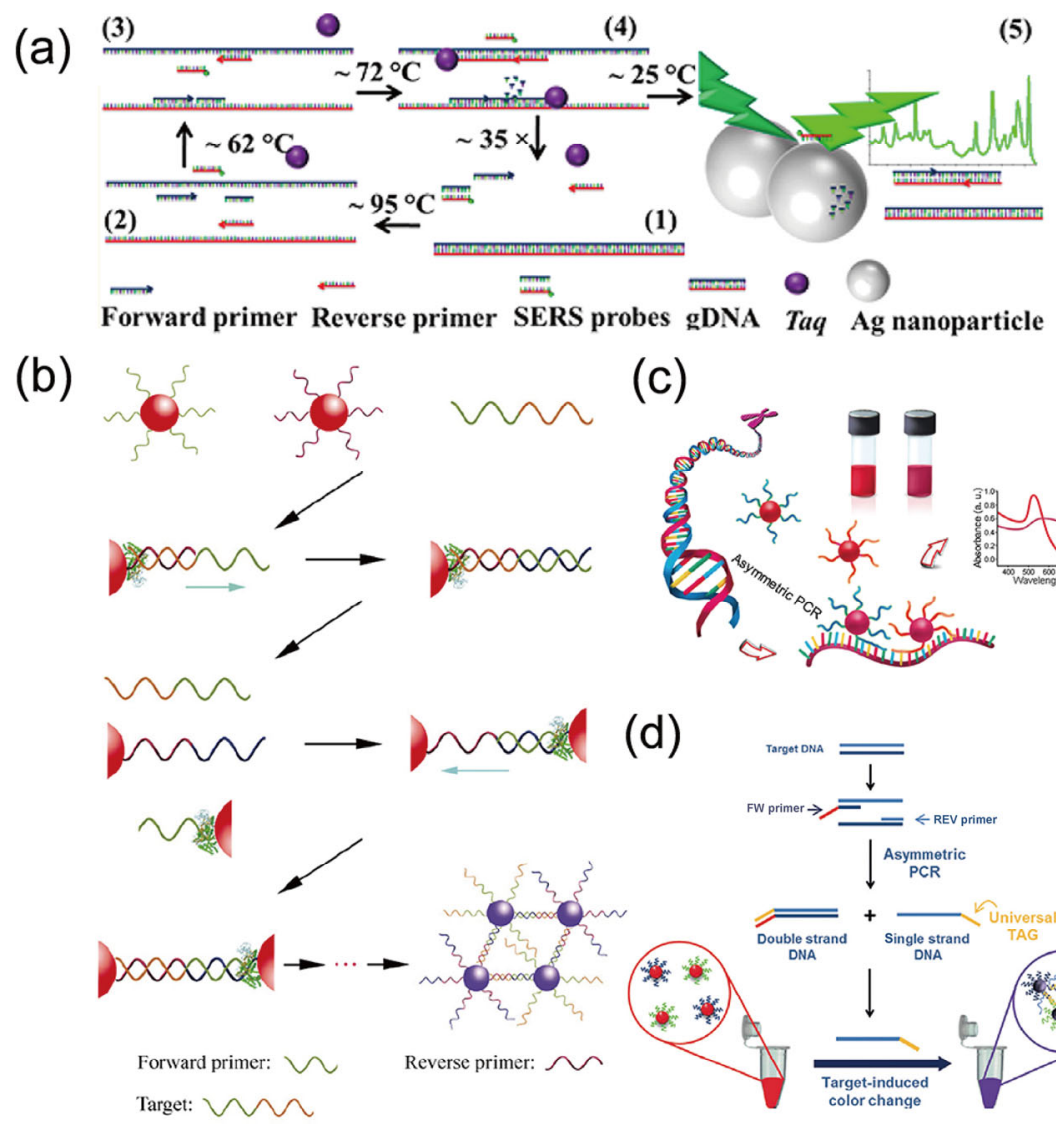

(c)
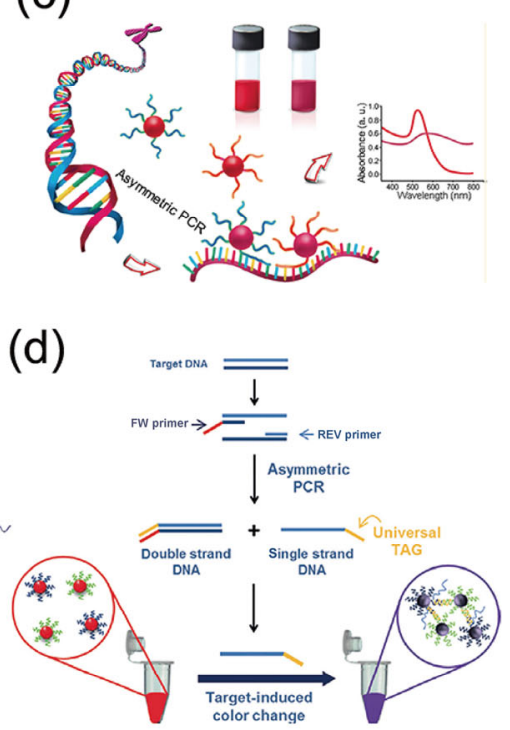

Figure 5 Various plasmonic implementations in PCR assay. (a) SERS-based PCR assay using SERS nanoparticle-conjugated primers (reproduced with permission from Ref. [116], (C) American Chemical Society 2013). (b) Colorimetric PCR using Au NP-conjugated primers (reproduced with permission from Ref. [117], (C) Springer Nature 2010). (c) Colorimetric Au NP labels for asymmetric PCR (reproduced with permission from Ref. [118], C American Chemical Society 2012). (d) Asymmetric PCR assay based on Au NP aggregation (reproduced with permission from Ref. [50], C Wiley-VCH 2016). 
Plasmonic NPs with vivid colors have been used as colorimetric labels for PCR, which eliminate the need of more expensive, less stable fluorescent dyes, and therefore enable rapid quantification of PCR results without the need of complex optical instrumentation. Wang et al. reported a colorimetric PCR assay where $\mathrm{Au}$ NPs were conjugated directly to the primers [117]. As shown in Fig. 5(b), the extension of Au NP primers during the PCR process triggered the aggregation of $\mathrm{Au}$ NPs. This provides a convenient solution for ultrasensitive, real-time detection of PCR products without the need of post staining. Liang et al. reported a colorimetric method for rapid quantification of asymmetric PCR products at picogram detection sensitivity by labeling the single-stranded products with oligo-functionalized Au NP probes (Fig. 5(c)) [118]. Pompa et al. demonstrated an improved version of colorimetric strategy, based on the controlled Au NP aggregation, for detection of as low as 0.01 zeptomole of HIV DNA against an excess of human genomic DNA [50]. In this method, asymmetric PCR was performed to yield predominant single-stranded products containing a universal tag sequence (yellow sequence in Fig. 5(d)). After completion of reaction, two sets of $\mathrm{Au}$ NPs functionalized with complementary oligonucleotide probes to different portions of the universal tag were added. In the presence of amplified targets, $\mathrm{Au}$ NPs were crosslinked by the universal tags, aggregated, and then color-shifted. The assay has been demonstrated for sensitive detection of HIV DNA with the naked eye, showing great promise for use in the POC settings. More recently, $\mathrm{Xu}$ et al. demonstrated that unmodified Au NPs could also be used for colorimetric detection of asymmetric PCR products by combining salt-induced nanoparticle aggregation assay [119]. In this design, PCR-produced single-stranded products protected Au NPs from aggregation in the presence of high salt concentration. In contrast, the absence of PCR products would induce nanoparticle agglomeration and a blue shift of resonance color. Using this assay, the LOD of viable emetic $B$. cereus was reported to be as low as $92 \mathrm{CFU} / \mathrm{mL}$ in $0.01 \mathrm{M}$ phosphate-buffered saline and $340 \mathrm{CFU} / \mathrm{mL}$ in milk. These Au NP based approaches greatly simplify the readout process, making PCR-based strategies possible for the POCT applications.
More recently, plasmonics-generated localized heat, a process known as photothermal conversion, has been utilized to speed up the rate of PCR assay. The concept of using plasmonic heating for PCR amplification was first demonstrated in 2012 by Roche et al [120]. In their study, $60 \mathrm{~nm}$ citrate-capped Au NPs were added directly to the PCR reaction mixture to a final concentration of $6.6 \mathrm{pM}$ (Fig. 6(a)). The $25 \mu \mathrm{L}$ reaction solution was then heated by a $532 \mathrm{~nm}(2.7 \mathrm{~W})$ continuous laser and cooled by active airflow. Rapid heating and cooling rates of $7.6^{\circ} \mathrm{C} / \mathrm{s}$ and $3.3^{\circ} \mathrm{C} / \mathrm{s}$ were obtained, respectively, allowing 30 cycles of amplification in less than 10 minutes. The ramp rate of the plasmonic thermocycler was faster than all commercially available PCR instruments at that time $\left(1-6{ }^{\circ} \mathrm{C} / \mathrm{s}\right)$. Later, Lee et al. reported a novel ultrafast photonic PCR through plasmonic photothermal heating of thin Au films (120 nm thick) illuminated by low-cost LEDs (450 nm wavelength) [121]. As shown in Fig. 6(b), this thin film-based thermocycler significantly improved the heating and cooling rates to 12.8 and $6.6^{\circ} \mathrm{C} / \mathrm{s}$, respectively, capable of completing 30 cycles within 5 minutes for a $10 \mu \mathrm{L}$ reaction volume. However, one potential limitation of plasmonic thin film is that the heating range is limited to approximately $100 \mathrm{~nm}$ from the gold surface, limiting its applications toward bulk solutions. This however has been improved later by using two layers of plasmonic films to form an optical cavity as thick as $750 \mu \mathrm{m}$, within which the PCR mixture experienced a more uniform temperature distribution across the cavity [113]. By optimizing the film thickness of top and bottom plasmonic films, ultrafast 30 thermal cycles from 68 (annealing/extension) to $94{ }^{\circ} \mathrm{C}$ (denaturation) were achieved within 4-10 $\mathrm{min}$ for reaction volume from $1.3-10 \mu \mathrm{L}$. Nucleic acids (c-MET cDNA) at concentrations as low as $10^{-8} \mathrm{ng} / \mu \mathrm{L}$ (two copies per $\mu \mathrm{L}$ ) were successfully amplified. Alternatively, Weizmann et al. demonstrated the use of gold bipyramid nanoparticles (Au BPs) as photothermal convertors for ultrafast real-time DNA amplification analysis. The benefits of using suspended nanoparticles as localized heating source are twofold: it not only allows more rapid and uniform heating, but also provides the clearance for real-time optical detection of the PCR products (Fig. 6(c)) [122]. The plasmon-resonant wavelength of $\mathrm{Au}$ BPs was chosen 
(a)

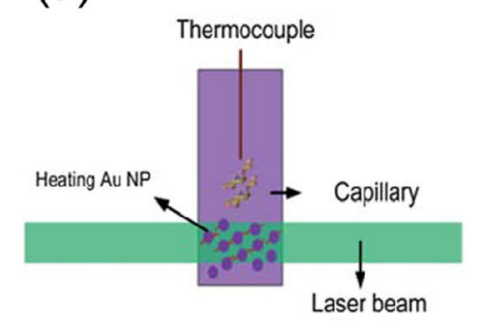

(b)

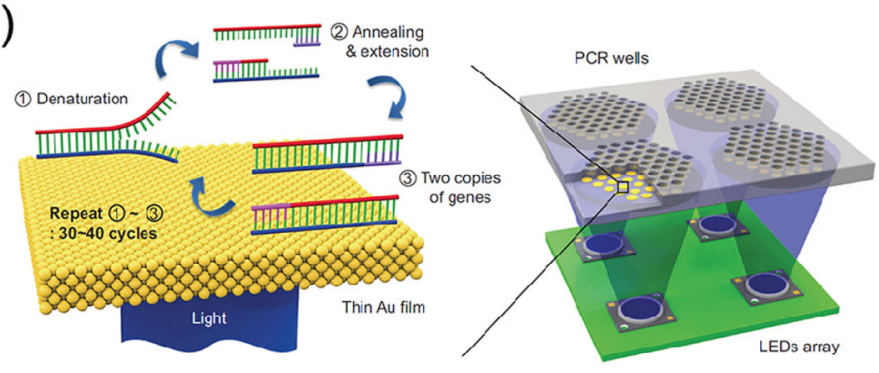

(c)

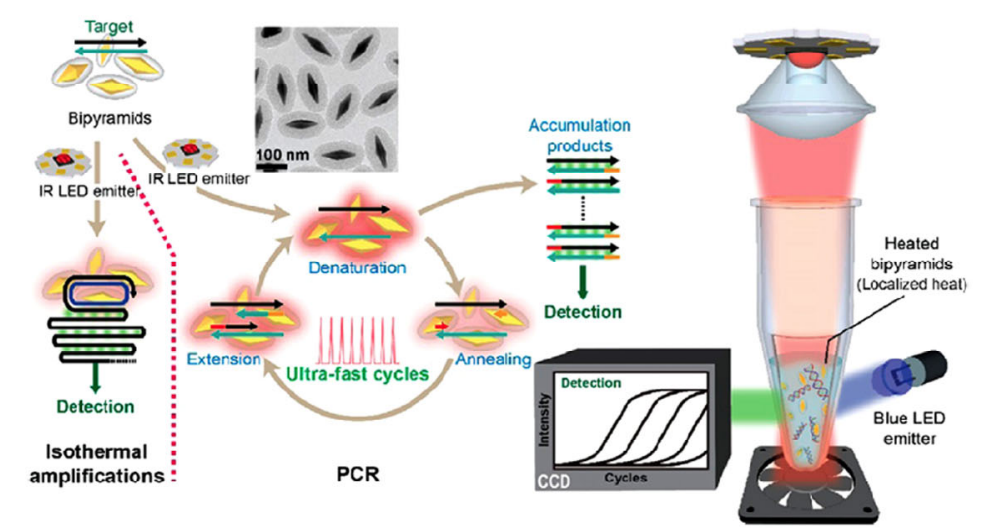

Figure 6 Various ultrafast plasmonic PCR platforms. (a) Schematic of plasmonic PCR using Au NPs as the photothermal converter (reproduced with permission from Ref. [120], (C) Royal Society of Chemistry 2012). (b) Ultrafast photonic PCR using gold film as photothermal converter and inexpensive LEDs as light source (reproduced with permission from Ref. [121], (C) Springer Nature 2015). (c) Photothermal PCR using gold bipyramid nanoparticles (Au BPs) (reproduced with permission from Ref. [122], (C) American Chemical Society 2017).

to be located in the near infrared $(846 \mathrm{~nm})$ region which was far away from the excitation wavelengths of the routine intercalating dyes. The platform was applied for both real-time PCR (40 cycles within $7.5 \mathrm{~min}$ for $5 \mu \mathrm{L}$ volume, with a LOD of $1 \mathrm{pg} / \mu \mathrm{L}$ DNA) and isothermal rolling circle amplifications (RCA). Roche et al. demonstrated the fastest plasmonic PCR so far that achieved 30 cycles in 54 seconds using dualfunctional gold nanorods (Au NRs) as both heating source and refractive index sensor for real-time monitoring of PCR products [123].

\subsubsection{Plasmonics-enhanced isothermal DNA amplification}

Isothermal nucleic acid amplification is a simple process that amplifies nucleic acid sequences at a constant temperature. As an alternative to PCR, isothermal nucleic acid amplification methods have been widely used for biomolecular diagnosis, especially when complicated and bulky thermocyclers are not available. Generally, these reactions can be divided into two categories: 1) the enzyme-aided approaches, such as loop-mediated isothermal amplification (LAMP), rolling circle amplification (RCA), and cyclic enzymatic amplification (CEA), and 2) enzymefree approaches, predominantly DNA circuit-based amplifiers such as hybridization chain reaction (HCR) and catalytic hairpin assembly (CHA). Similar to conventional PCR, plasmonics has been frequently integrated with isothermal DNA amplification methods to improve detection sensitivity or simplify detection process.

Use of plasmonic nanoparticles as colorimetric reporters is a common strategy to enable sensitive and rapid readout with a simple instrument, or even with the naked eye. Au NP reporters have been used in many isothermal amplification methods including CEA [124-126], LAMP [127-129], RCA [130], HCR [131, 132], CHA [133], and other DNA machinery [134, 135]. For example, Liu et al. developed a colorimetric DNA biosensor through the use of a nicking endonuclease 
assisted Au NPs amplification [126]. The strategy provided the capability of recognizing long singlestranded oligonucleotides with single-base mismatch selectivity and a $10^{3}$-fold improvement in amplification (ca. 10 pm) (Fig. 7(a)). LAMP uses two or three sets of primers (including a forward inner primer (FIP), backward inner primer (BIP), and outer primers (F3 and B3)) and a polymerase to amplify target DNA sequence with excellent specificity at a constant temperature $\left(60-65^{\circ} \mathrm{C}\right)$ in $30-60 \mathrm{~min}$. The LAMP reaction is consisted of two stages, including the dumbbell structure-producing step and the cycling amplification step. Lee et al. developed an ultrasensitive colorimetric LAMP assay using carboxyl-modified Au NPs as detection labels (Fig. 7(b)) [127]. 11-mercaptoundecanoic acid-functionalized Au NPs first formed aggregation in the presence of magnesium ion $\left(\mathrm{Mg}^{2+}\right)$, an enzyme cofactor of LAMP, and then dissembled by increasing concentration of pyrophosphate ion $\left(\mathrm{P}_{2} \mathrm{O}_{7}^{4-}\right)$, a byproduct of the amplification process. A LOD of 200 copies DNA per $20 \mu \mathrm{L}$ reaction (or $17 \mathrm{aM}$ ) was reported. Wang et al. reported an enzyme-free colorimetric strategy to detect DNA using Au NP-integrated HCR amplification [131]. HCR is a process involving cascade recognition and hybridization between two sets of DNA hairpin molecules, leading to the formation of a long double helix that grows until the hairpins are exhausted. As shown in Fig. 7(c), in the absence of target DNA, the two sets of hairpins with auxiliary single-stranded toehold would adsorb on the surface of Au NPs and protect them from aggregation in high salt solution. In contrast, in the presence of target DNA and HCR reaction was triggered, the double-stranded amplification products were less stable on the Au NP surface without sticky toeholds, therefore resulting in aggregation. This simple strategy provided a LOD of $50 \mathrm{pM}$ for instrument detection and $100 \mathrm{pM}$ for naked eye detection. This format of strategies can be performed at room temperature and doesn't need to modify the Au NPs, which is very suitable for POCT applications.

In addition to plasmonic colorimetry, SPR is widely used for real-time quantification of isothermal amplification products. SPR has been implemented in LAMP [136], RCA [137], strand displacement amplification (SDA) [138], RNA transcription-based amplification [139], HCR [140-143], and many other nonenzymatic DNA circuit amplifiers [144-146].

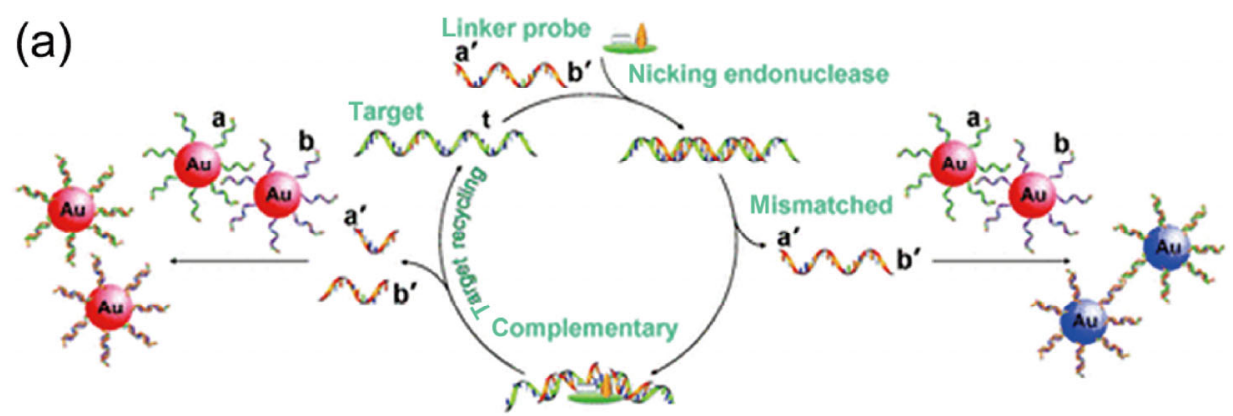

(b)

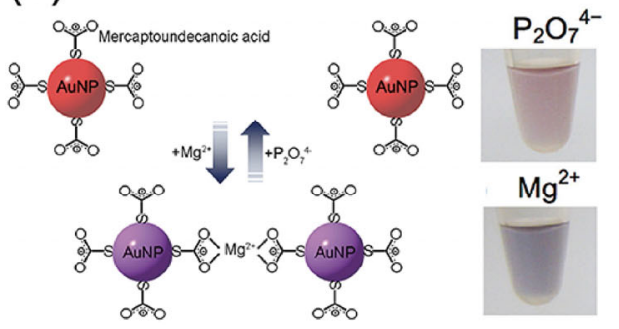

(c)

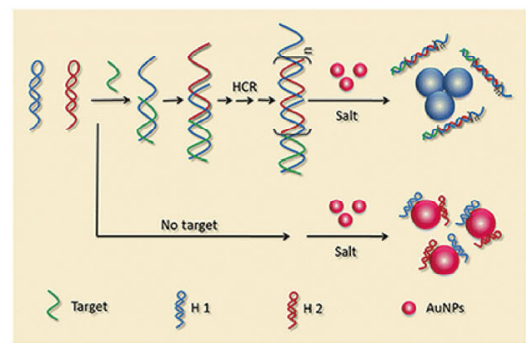

Figure 7 Plasmonic NPs as colorimetric labels for isothermal DNA amplification assays. (a) Nicking endonuclease assisted Au NP amplification for target DNA detection (reproduced with permission from Ref. [126], C) Wiley-VCH 2009). (b) Colorimetric LAMP assay using 11-mercaptoundecanoic acid functioned Au NPs as detection labels (reproduced with permission from Ref. [127], (C) WileyVCH 2014). (c) Enzyme-free detection of DNA using Au NP-based HCR amplification (reproduced with permission from Ref. [131], (C) American Chemical Society 2013). 
Strategies that can further enhance the SPR sensitivity include the use of Au NPs as plasmonic coupler or the design of a nonlinear amplification process. For example, Ding et al. reported a SPR biosensor for nonlinear HCR for the detection of two major transcript subtypes of PML/RAR $\alpha$, diagnostic biomarkers for acute promyelocytic leukemia (APL) [142]. As shown in Fig. 8(a), in the presence of target DNA, the cascade self-assembly process formed dendritic DNA structures instead of linear products generated by conventional $\mathrm{HCR}$, which led to a significant enhancement to the SPR signal.

SEF and SERS could also be powerful in the detection of isothermal amplification assays. Liu et al. demonstrated a strategy to perform HCR reaction directly on a nanostructured gold island film [147]. By labeling the nicking double helix products with fluorophores, the nanogold SEF substrate greatly enhanced the fluorescence emission of the HCR products (Fig. 8(b)). This plasmon-enhanced HCR (PE-HCR) was able to achieve a LOD of $0.043 \mathrm{fM}$ (1,300 molecules in $50 \mu \mathrm{L}$ of sample). Zhang et al. reported a sensitive RCA assay by performing surfaceinitiated RCA on a polycrystal gold electrode and further labeling the single-stranded products with
SERS tag-conjugated Au NPs (Fig. 8(c)) [148]. The Au NP assembly mediated by the DNA coils formed a SERS-active substrate for fingerprint detection of DNA as low as $10 \mathrm{pM}$, as well as for detection of single-base mismatch. Zhang et al. developed a hybrid two-step DNA amplification assay combing cross SDA and RCA with its end products quantified by SERS after hybridization with Raman probes [149].

\subsubsection{Plasmonic methods for DNA genotyping and sequencing}

The human genome contains nucleotide sequence variations at an average frequency of $0.1 \%$. Various forms of sequence variations have been identified and studied, including single nucleotide polymorphism (SNP), copy number variation (CNV), short tandem repeat, and many others. All contribute to the genomic diversity. Efficient detection of sequence variations would greatly enhance our understanding of the correlation between genotype and disease susceptibility, genetic epidemiology and population genetics, and improve diagnosis of infectious diseases by accurate discrimination between two similar disease strains with different virulence. Current strategies for sequence profiling including DNA genotyping and sequencing.

(a)

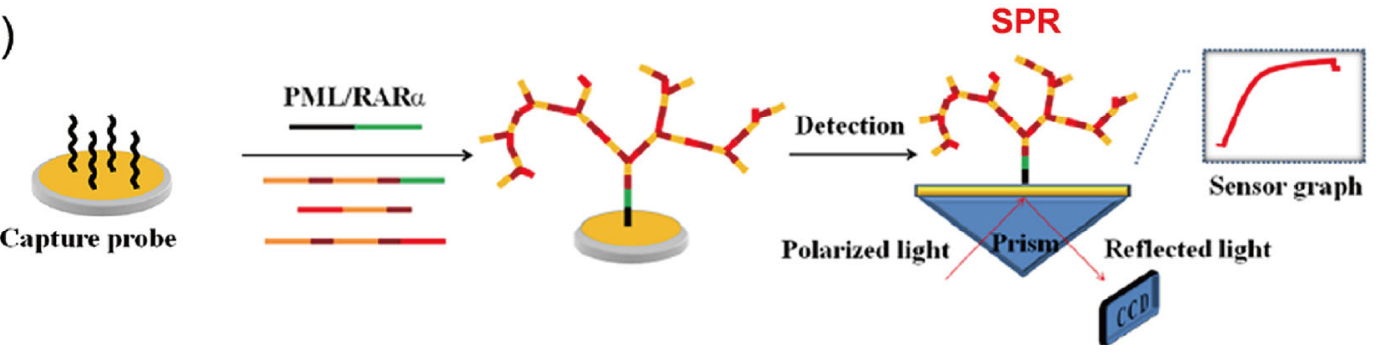

(b)

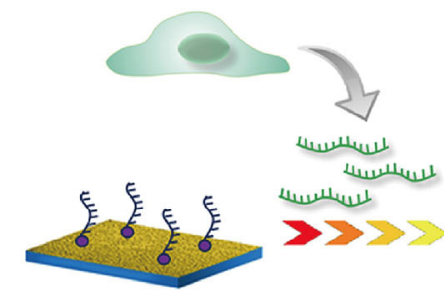

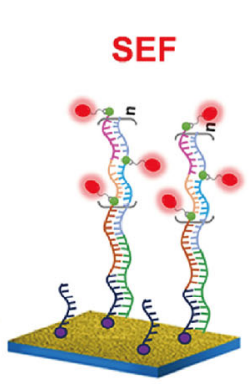

(c)

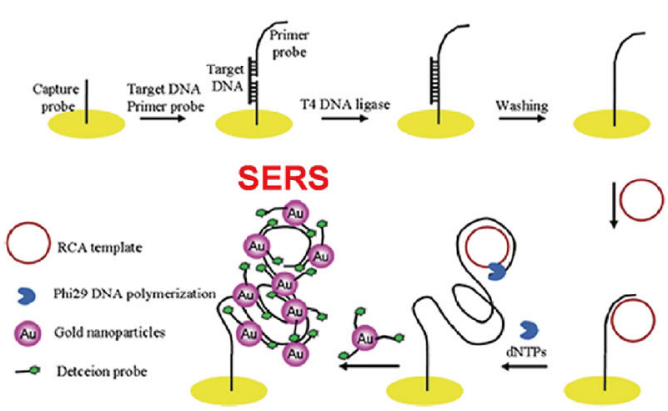

Figure 8 Surface-enhanced plasmonic technologies used in isothermal amplification methods. (a) Schematic illustration of the SPR-based non-linear HCR (reproduced with permission from Ref. [142], (C) Springer Nature 2017). (b) SEF-based HCR assay (reproduced with permission from Ref. [147], (C) American Chemical Society 2016). (c) SERS-based RCA assay for DNA detection (reproduced with permission from Ref. [148], (C) American Chemical Society 2010). 
The potential of using SPR sensors for DNA genotyping has been well demonstrated in the last decade $[150,151]$. The basic idea is to create a large array of ssDNA probes consisted of a library of complementary sequences targeted for different subtypes on a SPR sensor chip to screen unknown strains in a flow chamber. The hybridization of perfectly matched sequences leads to a shifted SPR signal that can be detected in each microarray spot. Such approach is sensitive, high throughput, and can be easily multiplexed. In addition, genotyping of SNP [152, 153], one of the most frequent genetic variations, is also feasible with SPR sensing. Ward et al. developed a DNA ligationbased SNP assay combing SPR sensing for SNP allele discrimination associated with venous thromboembolism (VTE) [154]. In their design, the capturing sequences were arrayed on a SPR chip, and target sequences were hybridized in the presence of a biotinylated detection probe and a DNA ligase (as shown in Fig. 9(a)). Ligation of the detection probes to perfectly matched capturing sequence was visualized by a subsequent localized enzymatic colorimetric reaction that led to a color change of the array spot enhanced by the SPR effect. Various approaches have

(a)

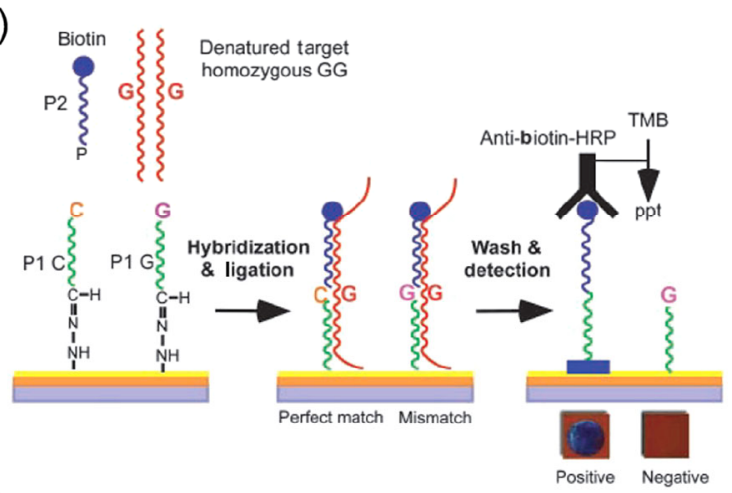

(b)

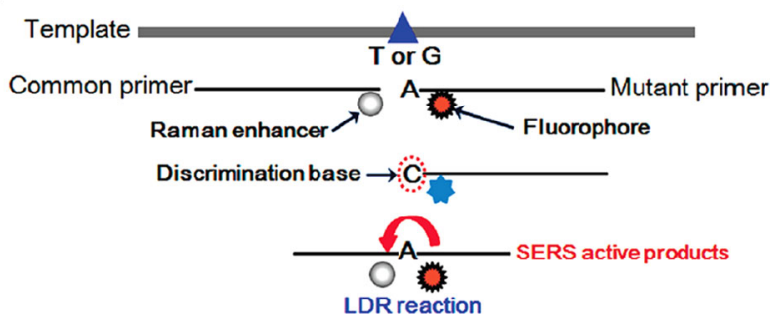

Figure 9 (a) SPR based SNP genotyping (reproduced with permission from Ref. [154], (C) National Academy of Sciences 2003) and (b) SERS for SNP genotyping, coupled with ligation chemistry (reproduced with permission from Ref. [157], (C) American Chemical Society 2010). been developed to enhance the SPR readout for SNP detection including the use of Au NPs as the enhancer [155] as demonstrated previously in protein detection.

SERS is an alternative method for DNA genotyping. Stevenson et al. reported DNA genotyping with SERS for multiplexed detection of up to 6 subtypes [156]. In this study, human papilloma virus (HPV) genotypes were first amplified by PCR with validated PCR primer sets and then hybridized with 6 different SERS tagconjugated ssDNA probes. After magnetic separation, SERS probes bound to the present subtypes were eluted and disposed onto silver NP substrates. Discrimination of different strain types was then achieved by identification of characteristic Raman resonance peaks in the SERS spectra. Batt et al. demonstrated a ligation detection reaction (LDR) coupled with SERS for multiplex SNP genotyping of KRAS oncogene alleles at $10 \mathrm{pM}$ detection sensitivity [157]. As shown in Fig. 9(b), the Raman tag and enhancer were conjugated to two separate ssDNA probes and brought together to generate SERS signals only when fully matched target templates were hybridized and ligated.

DNA sequencing on the other hand requires the detection of the base order of a nucleotide chain at the single-base resolution, which has imposed a significant challenge for both molecular assay technology and detection. DNA sequencing technologies have evolved from early Sanger sequencing to currently dominant next-generation sequencing (NGS) [158-161]. The advancement of nanotechnology is further changing the landscape of DNA sequencing by providing new alternatives with single-molecule sensitivity and real-time sequencing capability. Two of the leading third-generation sequencing technologies include nanopore sequencing and single-molecule, real-time sequencing (SMRT).

Solid-state nanopore platforms are being extensively utilized in the growing field of rapid DNA sequencing. The nanoscale pores provide a highly confined space within which single strand nucleic acid can be analyzed at high throughput through the changes of ionic [162] or tunneling currents [163]. SMRT $[164,165]$ on the other side still relies on optical detection of fluorescent events similar to NGS but aims to increase sequencing speed and throughput (Fig. 10). At the heart of the SMRT technology is a dense array of plasmonic 

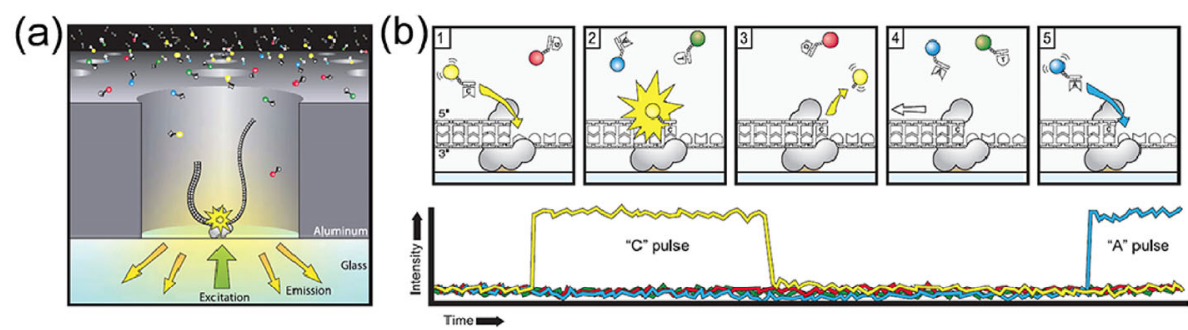

(c)
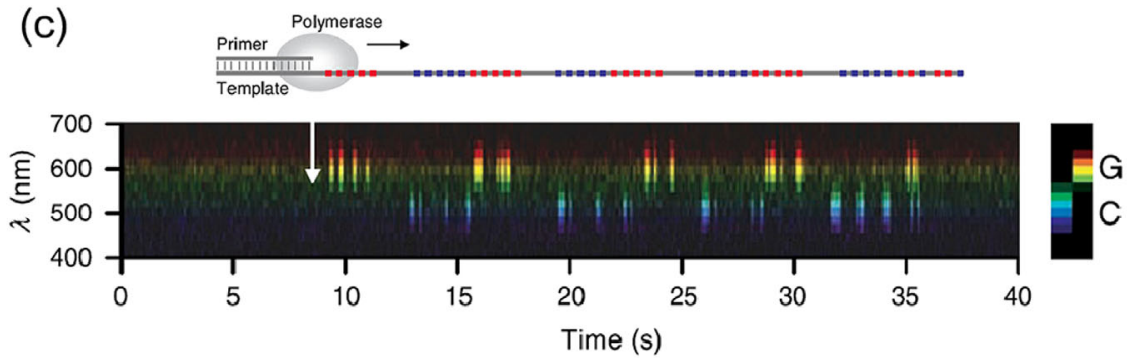

Figure 10 Schematic illustration of ZMW nanoarray for SMRT. (a) DNA polymerase was immobilized at the bottom of a ZMW and illuminated by laser light. (b) Fluorescence intensity raised at a specific time via DNA polymerase mediated incorporation of fluorophore-conjugated dNTPs. (c) Real-time observation of DNA synthesis by single-molecule spectroscopic measurement (reproduced with permission from Ref. [164], (C) Science 2009).

nanowells that work as zero-mode waveguides (ZMW) [166] for single-molecule imaging. In this regard, nanoplasmonics again has been playing a key role. ZMW utilizes the metal wall as the cladding and the nanohole as the core of the waveguide, which blocks the propagation of light into the waveguide above the cut-off wavelength (Fig. 10(a)). It then creates a highly confined illumination volume of the incident light in a $10-20 \mathrm{~nm}$ thickness at the bottom of each nanowell (zeptoliter volume), allowing single-molecule observation even at high concentrations. In SMRT, polymerase was immobilized at the bottom of ZMW, and the reversible incorporation of fluorescently labeled nucleotides on the template was monitored in real time by single-molecule spectroscopic measurement (Figs. 10(b) and 10(c)) [164, 165].

New plasmonic technologies for DNA sequencing are also emerging. Deckert et al. attempted to directly scan the sequence of a single RNA strand by using tip-enhanced Raman scattering (TERS) based on the characteristic Raman signatures of different nucleotides (Fig. 11(a)) [167]. The limitations are however the throughput and the lateral resolution to resolve single bases due to the relatively large size of scanning tips. Ju et al. proposed a different strategy of sequencing DNA using SERS [168]. Similar to fluorescently labeled nucleotides routinely used in NGS, they synthesized SERS tag-labeled reversible terminators by attaching an azido moiety $\left(\mathrm{N}_{3}\right)$, an intense Raman report, to the 3'-OH group of individual nucleotides. The cyclic incorporation and cleavage of Raman tag-labeled nucleotides into the DNA template was successfully detected by monitoring the SERS signal (Fig. 11(b)). By using 4 different Raman tags in future, fully automated DNA sequencing with a SERS detector is highly possible. Faramarzi et al. investigated the possibility of DNA sequencing with SPR sensing by simulating the different SPR shift of plasmonic substrates in the presence of different nucleotides when illuminated by ultraviolet (UV) light [169]. As shown in Fig. 11(c), the SPR properties of nanopore, bowtie, and bowtienanopore compound nanostructures consisted of a range of materials including graphene $(\mathrm{Gr})$, chromium $(\mathrm{Cr})$, aluminum $(\mathrm{Al})$, and, rhodium ( $\mathrm{Rh}$ ) were simulated by discrete dipole approximation (DDA). The bowtienanopore hybrid nanostructure made of $\mathrm{Cr}$ showed the highest selectivity to discriminate all four nucleotides (A, T, C, G) by displaying distinguished wavelength shifts for the four different nucleotides. Although the proposed method still requires thorough experimental validations, it may shed light on new methods for DNA sequencing in future. 
(a)
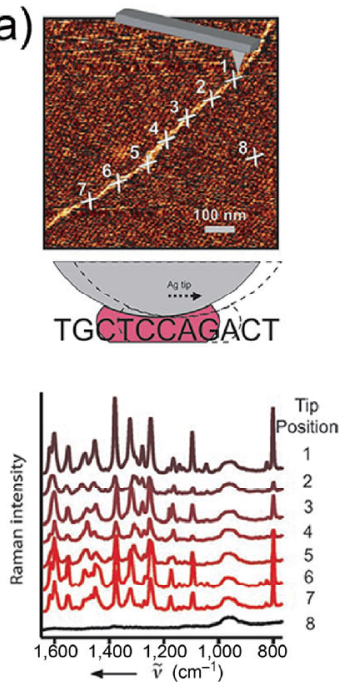

(b)
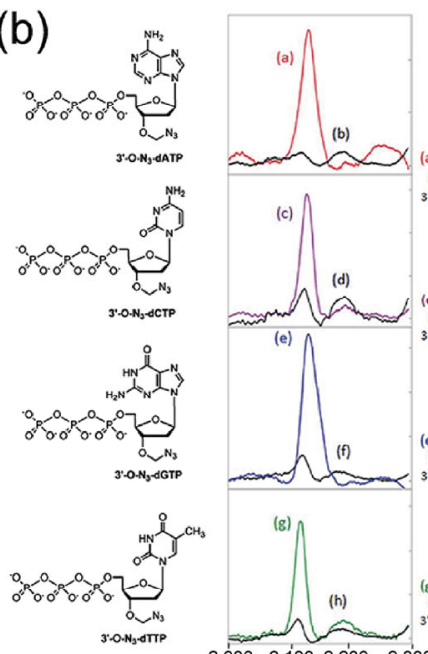

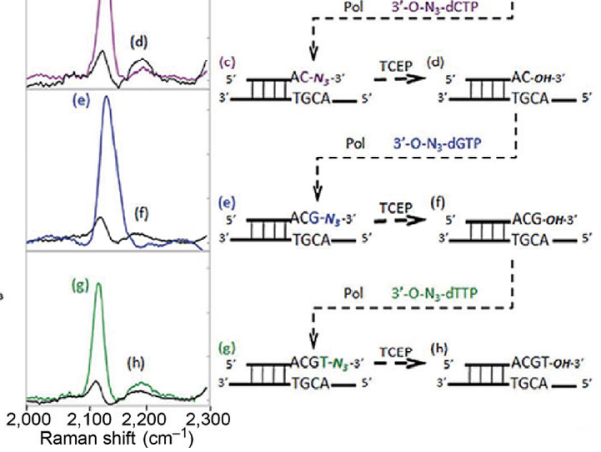

(c)

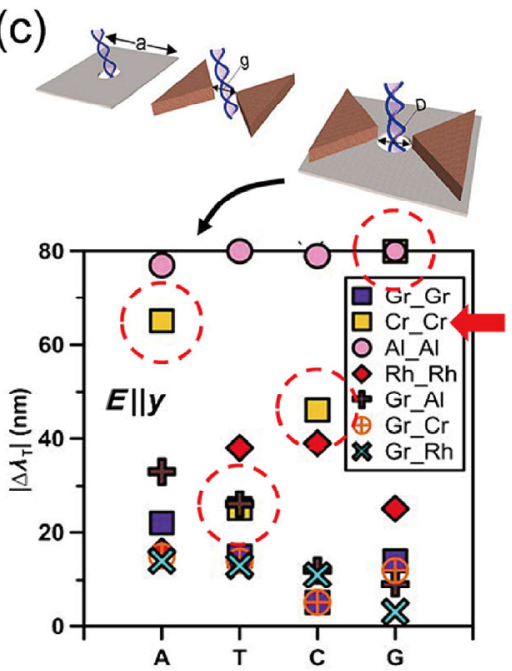

Figure 11 DNA sequencing by (a) scanning TERS (Reproduced with permission from Ref. [167], (c) Wiley-VCH 2008). (b) SERS (reproduced with permission from Ref. [168], (C) Royal Society of Chemistry 2014), and (c) SPR (reproduced with permission from Ref. [169], () Optical Society of America 2016).

\section{Integration with field-deployable assay platforms}

As the research of plasmonics-enhanced biomolecular analysis expands, the translation of laboratory plasmonic molecular assays into point-of-care tests (POCT) has become one of most promising applications. A complete POCT system would consist of minimally three components: the assay, chip, and reader. Here, the term "chip" refers to any simple, compact, field-deployable supporting matrix in which that the molecular assay can be contained and run to completion automatically. Two common "chips" are microfluidic and paper devices. The "reader" refers to miniature imaging and sensing devices that will be discussed in Section 5. They are portable, low-cost, easy-to-use, sensitive, accurate, and capable of rapid quantification of assay signals in the POC settings without the need of bulky benchtop instrumentation. Recent developments in microfluidics, smartphonebased microscopes, and wearable sensing technologies have paved the road for pushing plasmonic molecular assays from the lab to the patient side.

\subsection{Plasmonic microfluidic devices}

As one of the typical POCT platforms, microfluidics or lab-on-a-chip is widely acknowledged as an essential technology for POC applications due to the following merits: portability, cost-effectiveness, minimum sample requirement, high analytical throughput, and integrated function for assay automation. The synergistic integration of plasmonics and microfluidics (also termed as plasmofluidics) is emerging as a powerful biosensing strategy, and has become an essential step to turn benchtop plasmonic molecular assays into field-applicable tests.

The most common example of integrating nanoplasmonics with microfluidics is perhaps the microfluidic SPR sensor. By combining immunoassay chemistry (see Section 3.1.2) or DNA hybridization, microfluidic SPR devices allow for real-time, continuous, and parallel detection of a panel of targets simultaneously on a single chip. Microfluidic SPR sensor can be easily fabricated by binding conventional PDMS channels onto metal-coated SPR chip substrates. For example, Sonntag et al. developed a SPR-based (Au coated) microfluidic chip for detection of miRNA-93 [170]. This microfluidic SPR sensor enabled parallel analysis of three linear spot arrays, and the output signal was amplified by two different strategies, either by introduction of an enhancer antibody which bound to the RNA-DNA complex, or a Poly(A) polymerase that elongated the target miRNA by nucleic acid synthesis. Tokel et al. reported a microfluidic SPR 
device for the detection of Escherichia coli (E. coli) at concentrations ranging from $\sim 10^{5}$ to $3.2 \times 10^{7} \mathrm{CFUs} / \mathrm{mL}$ [171]. As shown in Fig. 12, the bacteria were captured by the antibodies immobilized in the microchannel with an Au-coated SPR chip, resulting in a local refractive index change due to the binding event.

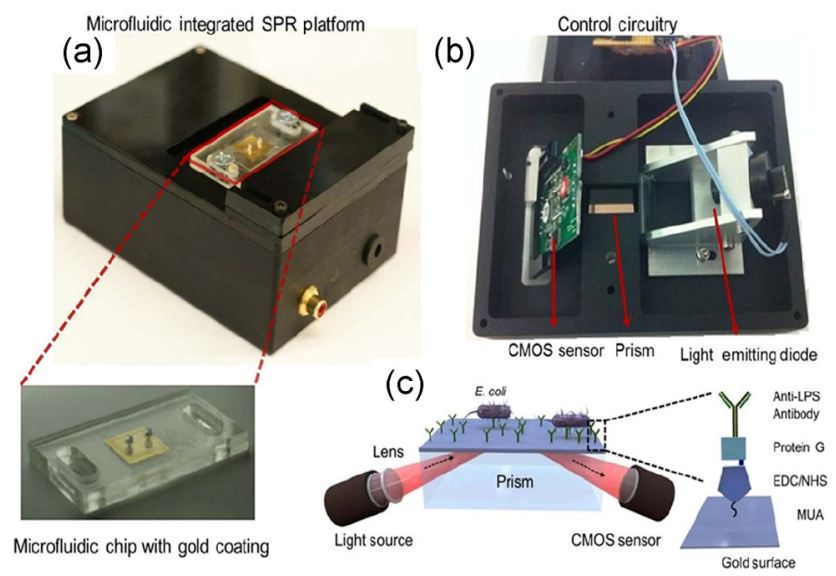

Figure 12 (a) Photograph of the SPR-based microfluidic system for pathogen detection, and 50-nm Au-coated glass substrate along with the microchip is shown below. (b) The electronic setup of this SPR detection system. (c) Schematic of the SPR detection mechanism. The refractive index was changed when the antibody captured the pathogen, providing a signature on the reflected light, which could be recorded by the sensing system. Reproduced with permission from Ref. [171], (C) Springer Nature 2015.
Applications of microfluidics for other plasmonic detections, such as LSPR [172], SERS [173-176], and SEF [177] have also been extensively studied. For example, Quidant et al. reported a parallel LSPR-based immunoassay chip for the real-time detection of AFP and PSA down to concentrations of $500 \mathrm{pg} / \mathrm{mL}$ in $50 \%$ human serum [172]. In this device, periodic gold nanorod (Au NR) arrays were fabricated on a glass substrate and separated into 8 independent microfluidic channels by PDMS with totally 32 multiplexed sensing sites on a single chip. Zhong et al. reported a SERSbased immunoassay in microfluidic chips for the detection of CEA [178]. Upon the bioconjugation of the target and SERS nanotags, the nanotags were magnetically trapped on a specific spot within the microfluidic channel, enabling an enrichment of "hot spots" for the SERS detection with a LOD of $0.1 \mathrm{pM}$. Choo et al. employed droplet microfluidic platform for SERS analysis of specific antigen fraction 1 (F1) in Yersinia pestis (Fig. 13) [174]. The polyclonal F1 antibody was modified on the surface of $40 \mathrm{~nm} \mathrm{Au}$ NPs, while the monoclonal F1 antibody was conjugated on the surface of a magnetic bead. Through the sequential droplet generation, transport, and merging, SERS nanotages and F1 antigens were mixed with magnetic beads to form sandwich immunocomplexes. (a)

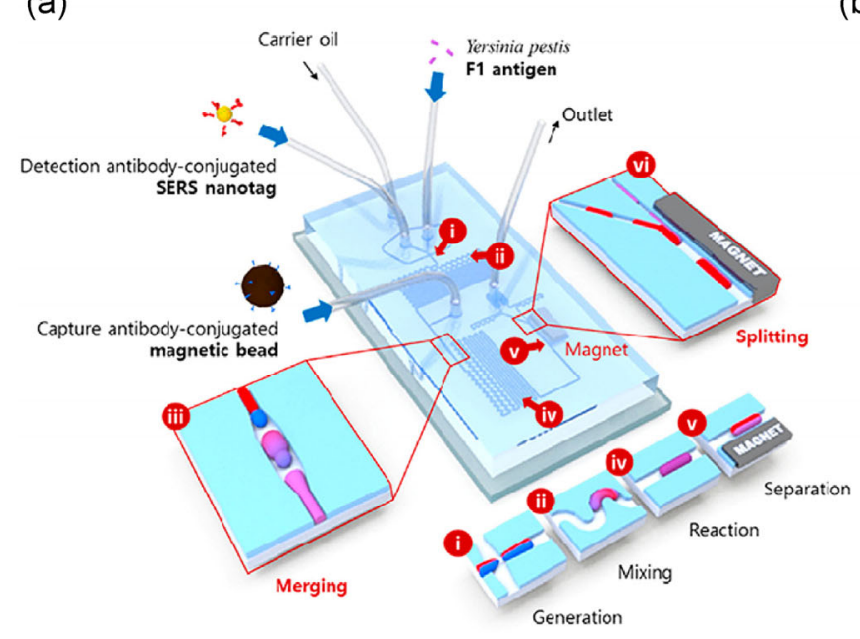

(b)
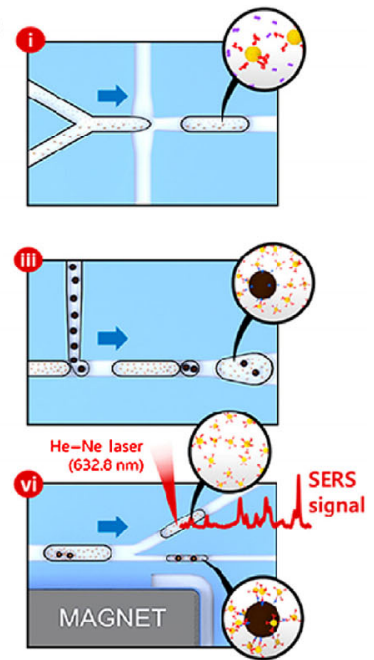

Figure 13 (a) Schematic design of the integrated SERS-based microdroplet platform: (i) droplet was generated from the shear force, (ii) first immunoreaction by droplet mixing, (iii) droplet merging with the magnetic beads (iv) second immunoreaction, (v) droplet splitting, and (vi) Raman detection of unbound SERS nanotags. (b) Extended images for (i) droplet generation, (iii) droplet merging for the formation of magnetic immunocomplexes, and (vi) droplet splitting. Reproduced with permission from Ref. [174], (C) American Chemical Society 2017. 
SERS detection was realized via magnetic droplets splitting with a LOD of $59.6 \mathrm{pg} / \mathrm{mL}$ (Fig. 13). This microfluidic chip is highly automated and integrated, though, precise flow operation is needed to obtain the results with good reproducibility.

\subsection{Plasmonic paper devices}

Over the last two decades, cellulose paper has been demonstrated as an ideal substrate to construct extremely low cost and disposable microfluidic devices for use in POC diagnostics. Paper devices are generally biocompatible, flexible, easy-to-fabricate, and support a broad range of assay chemistry with high surface areas $[179,180]$. Paper flow channels can be demarcated by the hydrophobic barriers, such as wax, printing ink, and photoresist [181]. Different from conventional microfluidics, paper-based microfluidics drive the sample fluid by capillary action without need of valve and pumps, which greatly simplifies the ways to operate these devices.

Plasmonic nanomaterials have been utilized to integrate with cellulose paper devices for POC sensing applications [182]. Lateral flow assays (LFAs), also commonly known as "dipstick tests", have been the predominant POC tests in the past decades [183].

Nowadays, LFAs are commercially available for a variety of diagnostic applications (e.g., pregnancy, HIV, infectious diseases, etc.). For example, in 2012, Au NP-based HIV LFA test using oral fluid was approved by Food and Drug Administration (FDA) [184]. LFAs are inexpensive, portability, rapid, and ideal for sample-to-answer applications without external instrumentation [185]. In a typical LFA, the biological fluid, such as blood, is added to the sample pad and mixed with pre-loaded detection probes (e.g., antibody/ DNA-conjugated Au NPs). Then the mobile phase will wick through the stationary phase by capillary action. When it passes through the test zone, the target will be captured by immobilized antibody or DNA probe. Au NP-conjugated detection antibody will be accumulated at the test line by forming the sandwich antibody-target-antibody complex.

To improve the analytical sensitivity and detection range of the Au NP-based LFA, Bischof et al. investigated the effect of different size of Au NPs $(30,60$, and $100 \mathrm{~nm}$ ) on the performance of LFA devices [186]. They found that the sensitivity was significantly dependent on the optical properties of Au NPs and their binding efficiency. The larger size Au NPs (100 nm) which possessed larger excitation cross section and higher binding affinity improved the detection sensitivity by 256-fold and expanded the detection range from $3 \log$ to $6 \log$. Gehrke et al. have investigated other plasmonic NPs than Au NPs as labeling agents for LFA [176]. Using resonant silver nanoplates, they developed multiplexed LFAs that could simultaneously detect dengue virus (DENV), yellow fever virus (YFV), and Ebola virus (Zaire strain, ZEBOV) with a LOD of $150 \mathrm{ng} / \mathrm{mL}$ for viral protein biomarkers of each disease. Xia et al. used dualfunctional Au NP-Pt core-shell nanoparticles (Au@Pt NPs) as LFA labels which produced more sensitive color change catalyzed by the Pt layers. The Au@Pt NP imbedded LFA assay showed greatly enhanced detection sensitivity by 2 orders of magnitude compared to conventional Au NP probes [187]. Although LFA is currently still considered a simple yes/no diagnostic test, commercialization of above demonstrated ultrasensitive plasmonic nanoprobes in LFA is currently on the way. For example, gold nanoshellenabled LFA with 20-fold increases in sensitivity is now available via nanoComposix [188]. There is a reason to believe that the performance of LFA will be continuously improved to be competitive to other molecular assays in future.

Paper-based SERS sensors provide a new option to perform Raman measurement in the POC settings. Plasmonic NPs can be integrated with cellulose fibers through seeded growth, adsorption, self-assemble, or in situ synthesis [189]. To date, various clinical samples including serum, tear, and whole blood have been detected through SERS on the paper substrates, which show great potential to be used for POC diagnostics [190, 191]. For example, Wu et al. reported a paperbased SERS assay for detection of Neuron-Specific Enolase (NSE) in blood plasma with a LOD of $0.86 \mathrm{ng} / \mathrm{mL}$ [191]. Schifferli et al. reported a gold nanostar (Au NS)-based LFA platform (Fig. 14), with the SERS limit of detection of $0.72 \mathrm{ng} / \mathrm{mL}$ of ZIKV NS1 and $7.67 \mathrm{ng} / \mathrm{mL}$ of DENV NS1 [192]. 


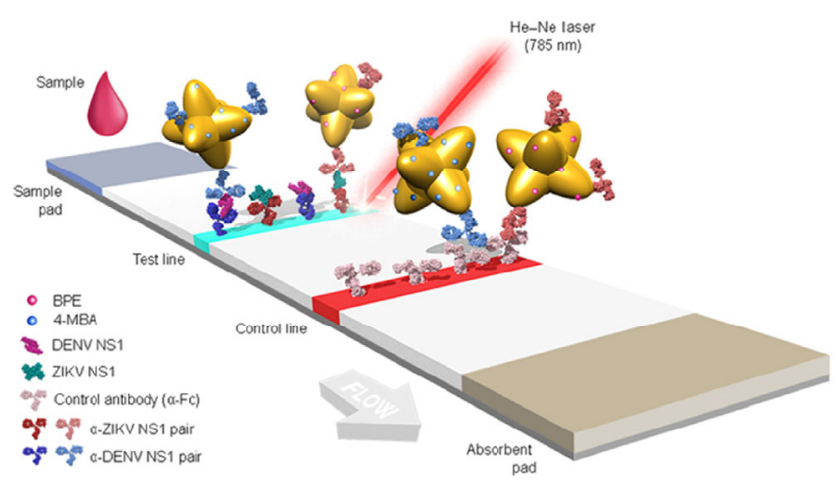

Figure 14 Schematic of dipstick sandwich immunoassay. Sandwiches formed by each antibody pair, NS1 and Au NS-Ab conjugate, for both ZIKV and DENV NS1, at the test line. Reproduced with permission from Ref. [192], (C) American Chemical Society 2017.

\section{Integration with portable readout devices}

\subsection{Miniature imaging devices}

Imaging and sensing tools are indispensable for biomedical detection and analysis. The field of optical microscopy is undergoing a profound transformation. The price and size of image sensors, light sources, and optical components have been significantly reduced. Device fabrication and prototyping is becoming increasingly inexpensive and faster than ever with 3D printing. With these trends, various cost-effective, field-portable, and miniature imaging and sensing tools are emerging, including lens-based [193-195] or lens-free on-chip microscopes [196], miniature fluorescence microscopes [197], and optical fiber microscopes [198]. Plasmonic sensing and measurement can now be readily performed on palm-size devices, providing a timely solution for individuals to monitor personalized tests in a real-time and continuous fashion. For example, Ozcan and Altug et al. developed a handheld plasmonic biosensor by coupling plasmonic Au nanohole arrays with lens-free on-chip holographic imaging system for screening of biomolecular binding events for POC use (Fig. 15) [199, 200]. This lightweight device utilized a CMOS imager chip to record the diffraction patterns (holograms) of the plasmonic microarrays excited by low-cost LEDs, with a minimum detectable refractive index change of $\sim 4 \times 10^{-3}$ RIU. More recently, a machine learning-based computational sensing framework was also developed, which could
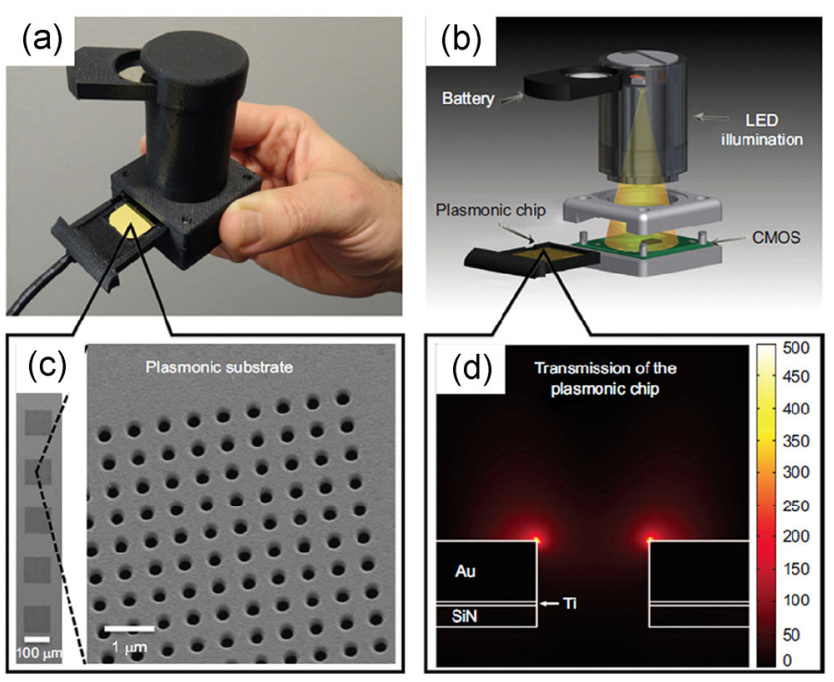

Figure 15 Handheld plasmonic biosensor based on lens-free imaging and Au nanohole array. (a) Photographs of the portable plasmonic reader device. (b) Schematic of the computational lens-free imaging system. (c) SEM image of the Au nanohole array. (d) FDTD simulation of the near-field intensity enhancement distribution for the nanohole array. Reproduced with permission from Ref. [199], (C) Springer Nature 2014.

be used to help select the most sensitive illumination wavelengths for designing cost-effective plasmonic reader devices [201]. Mirkin et al. reported a microarray format scanometric assay for the detection of protein and DNA. This strategy is based on the use of DNA microarray on glass slides and SNA-AuNPs to capture target DNA in a sandwich format [202]. Silver enhancement was further applied to enhance the signal via increasing the scattering intensity of assay spots. In 1997, this system was approved by FDA and now has been commercialized by Verigene System [203].

\subsection{Smartphone microscopes}

Roughly after four decades since the introduction of the first mobile phone, the global mobile phone subscription number has already reached more than 7 billion in 2017, and nearly $80 \%$ of these mobile phones are being used in developing countries [204]. Furthermore, mobile phones have been experiencing massive advances in their carried-on optical camera system, processing power, global positioning system (GPS), and wireless internet network, which all facilitate the use of such platforms for advanced biomedical measurements [205-208]. Various smartphone-based platforms have been explored for monitoring and 
diagnosis of diseases utilizing imaging modalities ranging from bright field [208, 209], scattering [210], to fluorescence [211]. Integrated with disposable assay chips, these field-portable and wirelessly connected devices provide a unique all-in-one platform for detection, data analysis, and remote reporting without the need of external computers.

Smartphone-based fluorescence microscopy is one of the predominant methods of smartphone sensing. The detection sensitivity of smartphone fluorescence microscopes has been significantly improved in the past few years, enabling detection of single nanoparticles as small as $100 \mathrm{~nm}$ [211], individual viruses [212], and single DNA molecules [213]. Recently, we have demonstrated a strategy to further improve fluorescence signals by implementing SEF in the smartphone microscopy [204]. As shown in Fig. 16, this smartphonebased SEF microscope used a silver thin film as fluorescence enhancement substrate and a glass hemisphere coupler to deliver the excitation light to the metal surface to generate SPPs. By optimizing the film thickness, spacer distance, illumination angle, and excitation polarization, a maximum fluorescence enhancement of $\sim 10$ fold was achieved, enabling detection of single fluorescent particles as small as

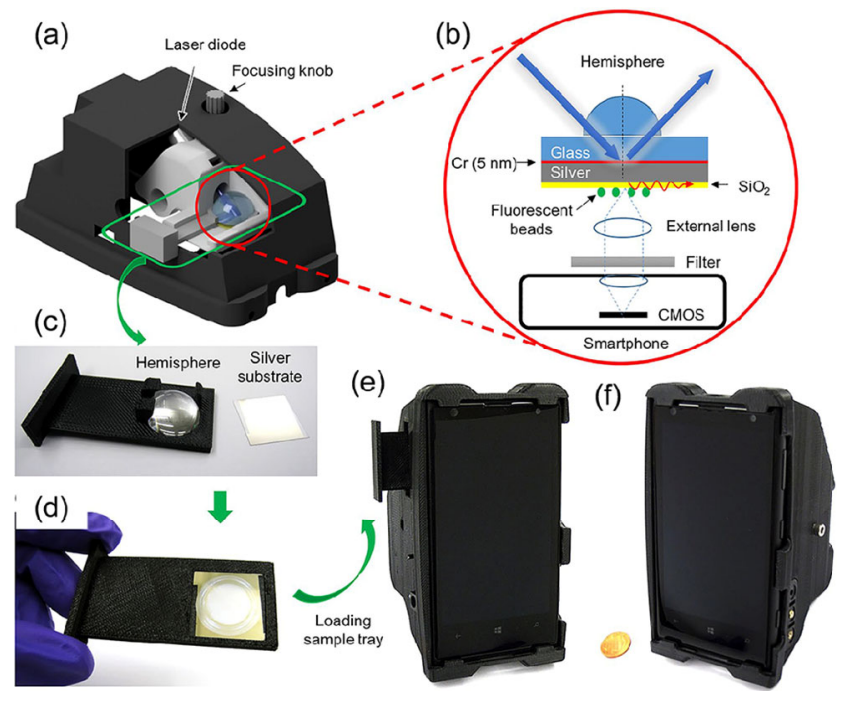

Figure 16 Plasmonics-enhanced smartphone fluorescence microscopy. (a) and (b) Schematic illustration of the smartphone attachment and illumination optical path, respectively. (c) and (d) Photographs of plasmonic silver substrate placed in the sample holders. (e) and (f) Photographs of the actual plasmonics-enahnced smartphone fluorescence microscope. Reproduced with permission from Ref. [204], C Springer Nature 2017.
$50 \mathrm{~nm}$ in diameter. A sensitivity limit of $\sim 80$ fluorophores per diffraction-limited spot was estimated by using fluorescently labeled DNA origami nanobeads as brightness standards.

Smartphones have also been employed as optical readers for various types of plasmon-resonant sensors such as coupling sensors, SPR, and LSPR sensors for use in the POC settings. Using its sensitive bright-field imaging mode, smartphones can be turned into miniature photometers for quantification of light absorbance of coupling-based colorimetric assays [214-216]. Moreover, a smartphone-based LFA assay reader has recently been commercialize by Cellmix [217]. To further improve the detection accuracy of colorimetric assays, ratiometric intensity quantification using two different color LEDs was demonstrated in a recent example [218]. This enabled the smartphone device to detect as low as $\sim 3.5 \mathrm{ppb}$ level of mercury contamination in water using an aptamer Au NP-based colorimetric assay. Filippini et al. demonstrated the first smartphone-based SPR refractive index sensor which is integrated with a microfluidic SPR sensor chip [219]. The smartphone camera was used to monitor the intensity change of the SPR signal after being reflected from the $\mathrm{Au}$ thin film. A LOD of $0.1 \mu \mathrm{g} / \mathrm{mL}$ of $\beta_{2}$ microglobulin $\left(\beta_{2} \mathrm{M}\right)$ was demonstrated. Since then, various SPR refractive index sensing substrates have been coupled with smartphones, including optical fiber sensors [220, 221], gratings [222], and plasmonic nanoarrays [223, 224]. For example, Wang et al. developed a grating-based SPR and a smartphone sensing system for lipopolysaccharides (LPS) detection with a LOD of $32.5 \mathrm{ng} / \mathrm{mL}$ in water [222]. This SPR sensing system relied on the smartphone's flash light as illumination source and an Au grating or compact disk (CD) as the spectral dispersive unit. Liu et al. demonstrated a smartphone-based SPR imaging platform (SPRi) for colorimetric biochemical sensing by combining a nano Lycurgus cup array (Fig. 17(a)) [223]. The optical absorbance of the colorimetric biochemical assay was enhanced by matching the SPR wavelength of the chip with the chromophore's absorbance peak wavelength. Such a sensing mechanism improved the LOD of BSA to $0.01 \mathrm{mg} / \mathrm{mL}$. Dana et al. demonstrated another smartphone-based SPRi platform using metal-coated 
(a)

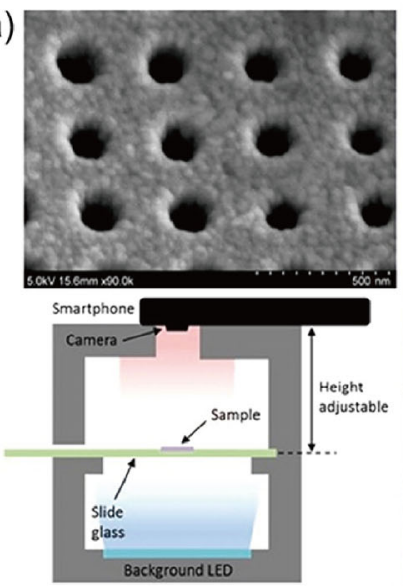

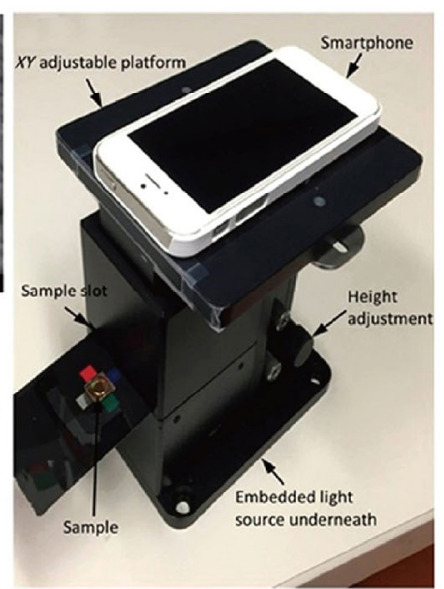

(b)

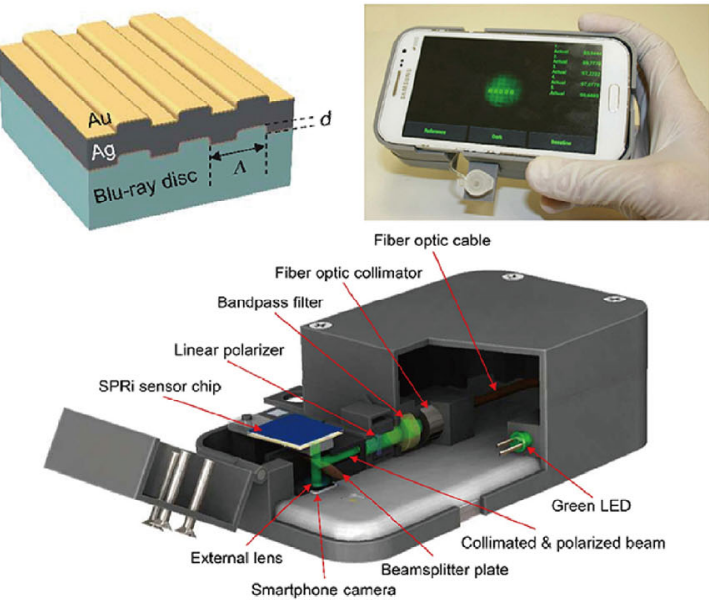

Figure 17 Smartphone-based SPR sensing platforms. (a) Smartphone-based SPR sensing platform using a nano Lycurgus cup array (nano LCA) (reproduced with permission from Ref. [223], (c) American Chemical Society 2017). (b) Au grating-enabled smartphone SPR sensor (reproduced with permission from Ref. [224], (C) Elsevier 2017).

Blu-ray discs as sensing substrate (Fig. 17(b)) [224]. A compact integrated imaging platform was developed using $3 \mathrm{D}$ printing, and refractive index sensitivity as low as $4.12 \times 10^{-5}$ RIU was demonstrated.

\subsection{Wearable and flexible sensors}

Wearable sensor technologies have been widely explored in the past few years for offering a comfortable and convenient way to continuously monitor an individual's physiological activities [225]. ultrathinness, light weight, and easy integration with clothes [226], wristband [227], and even artificial skin [228]. The coupling of plasmonic components on flexible, stretchable, and biocompatible substrates has attracted great attentions for wearable devices due to the unique optical sensing capability enabled by plasmonics. For instance, Song et al. fabricated a highly stretchable Au nanodisk array on a PDMS substrate using a simple transfer printing method. Its plasmonic resonance could be greatly shifted by stretching this flexible device, providing promising potential for use as soft optical sensors [229]. Zhou et al. developed a wearable colorimetric device based on LSPR nanostructures of nanowire clusters. This flexible plasmonic film could act as a humidity sensor to monitor sport sweating conditions, showing a sensing threshold of $0.028 \mathrm{mg} / \mathrm{cm}^{2}$, and the sensor was demonstrated to be very robust for accurate measurement against various wearing conditions (such as bending, twisting, and stretching of the device) [230].

Graphene-based plasmonics is of particular interest in the field of wearable devices, partially because of its tunability and potentially low damping rate [231]. In a recent work, Xia et al. developed a transparent plasmonic film based on graphene/insulator stacks [232]. Compared with single-layer graphene, this multiple graphene layer-based sensor effectively enhanced the plasmonic resonance frequency and magnitude sensitivity of carrier concentration. Implement graphene materials in wearable devices would also enable sensitive SERS detection. Compared with traditional metallic SERS substrates, hybrid graphene-metal NP substrates have shown as much higher SERS activity. Nam et al. developed a 3D crumpled graphene-Au NPs hybrid nanoplasmonic structures for amplified SERS sensing. This 3D hybrid structure can be easily generated on a PS substrate by heat treatment alone and shows higher Raman enhancement of at least an order magnitude than that of conventional flat graphene-Au NPs structures (Fig. 18) [233].

\section{Limitations and challenges}

Plasmonics-based POC molecular diagnostics has been well demonstrated as a cost-effective, sensitive, and specific detection strategy. The results of plasmonic assays can usually be quantified with field-portable reader devices or even the naked eye. These approaches 
(a)

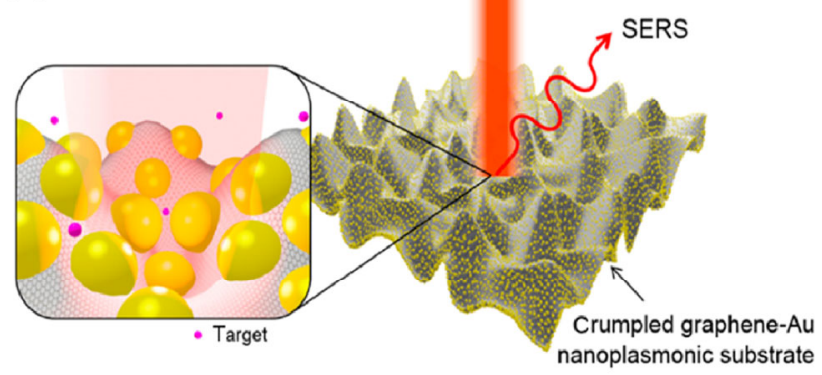

(b)

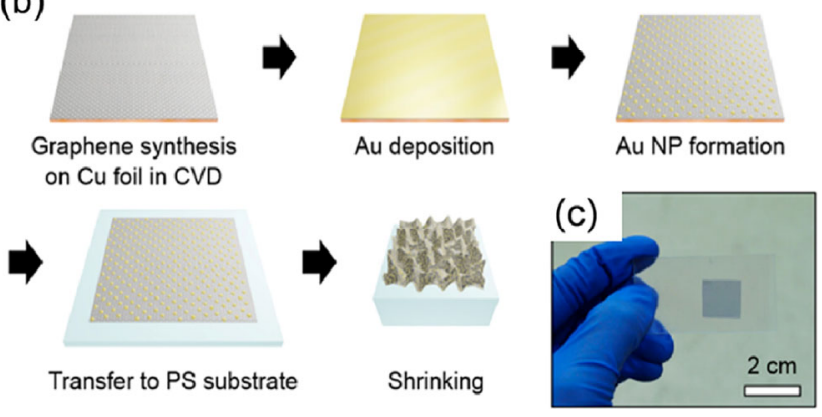

Figure 18 (a) Schematic illustration of 3D crumpled graphene-Au NPs hybrid structure for SERS sensing. (b) Process chart for sensor fabrication. (c) The photo of the 3D crumpled graphene-Au NPs hybrid nanoplasmonic structure (square-shaped dark region). Reproduced with permission from Ref. [233], (C) American Chemical Society 2015.

are therefore ideal for use in resource-limited settings, where rapid, cost-effective, portable, and reliable detection is highly desired. Despite that, few plasmonicsbased diagnostic techniques have been used in clinical diagnostic laboratories or commercialized so far. Lateral flow immunoassays based on Au NPs and single-molecule DNA sequencing based on plasmonic nanowell substrates (SMRT) are among the few successful examples. From the translational perspective, plasmonic molecular assays are still at their infancy stage although being successful in laboratory demonstrations. There remains a long way to go before taking those novel approaches from the lab bench to real clinical applications. In particular, for POC applications, there are several limitations and challenges of plasmonic molecular assays that need to be addressed before practical implementations.

\subsection{Sample preparation}

POC applications are different from laboratory testing where dedicated benchtop instrumentation is available.
This require molecular detection methods intended for POC use to be simple, self-contained, automated, and standalone. Conventional bioassays such as ELISA however require multiple washing and separation steps, making the detection process labor intensive, time consuming, and sometimes more susceptible to operational errors. Plasmonic technologies have significantly simplified the assay format in this regard. In the past, implementation of nanoplasmonics in bioanalysis enables the development of numerous label-free methods such as SPR and SERS, as well as washing-free protocols such colorimetric Au NP assays and turn-on fluorescence probes. This has reduced or completely eliminated the washing, purification, and separation steps in many diagnostic assays to allow continuous operations.

What has not been intensively explored yet is however the application of plasmonic technologies to facilitate sample preparation. Sample preparation refers to any necessary processing steps need to be taken for a raw biospecimen such as human whole blood before a molecular assay can be performed. Typical sample preparation steps may include cell lysis, DNA extraction, DNA fragmentation (for NGS), protein separation, purification, concentration, or a combination of several of them. Nanomaterials due to their large surface areas will be excellent candidates to promote molecular binding, targeting, and separation. Plasmonic nanostructures on the other hand would bring in new opportunities for rapid sample preparation schemes such as photothermal lysis of cells or bacteria due to their unique optical properties. Therefore, development of plasmonics-enhanced sample preparation methods will be likely a new focus for POC diagnostics, which will eventually lead to fully automated "sample-in, result-out" detection platforms.

\subsection{Uniform, cost-effective plasmonic substrates}

While the price of colloidal plasmonic nanoparticle solutions has been significantly reduced due to the tremendous improvement of synthetic methods in the past decades (e.g., > 95\% yield), a piece of millimetersize plasmonic substrate containing high density or well organized nanostructures could still be costly. Even a commercially available metal thin film-based SPR chip (10 mm $\times 10 \mathrm{~mm}$ size) still costs a few dollars 
per piece. More complicated nanostructured substrates requiring clean room-based nanofabrication would expect to cost even more. On the other side, bottom-up wet chemical synthetic approaches are economic alternatives to more expensive top-down fabrication methods. However, plasmonic enhancement substrates prepared by bottom-up methods (e.g., self-assembled monolayer nanocolloidal films or nanoporous metallic substrates) are frequently subject to less control on the size and distribution of plasmonic enhancement hot spots, ununiform signal enhancement across the substrate, chip-to-chip variation, and therefore low reproducibility for quantitative measurements. Therefore, there is a growing need for nanomaterials quality control for various applications, but only few standard quality control methods have been developed to date. Many existing nanoanalysis methods such as particle size analysis and optical spectroscopic measurement are limited by throughout and scalability, and are not suitable for continuous monitoring. Significant more efforts are thus needed in the area to develop scalable, in-line sensing technologies to assure the quality of nanomaterial production in a real-time fashion.

With the recent advance of 3D printing technology, it however provides a new route for cheap fabrication of functional plasmonic substrates with better control over the product quality. Due to the rapid improvement of 3D printing resolution, plasmonic substrates can be potentially prepared by either direct printing of nanoparticle-loaded ink or creating a high-resolution 3D-printed template for subsequent metal deposition [234, 234]. Nevertheless, 3D printing-based fabrication of plasmonic substrates is still at its infancy, and more research effects should be devoted to this area to develop a lower-cost, more uniform, and scalable fabrication solution to highly demanded plasmonic materials and substrates.

\subsection{Field testing and clinical validation}

The increase in awareness of human health and preventive diagnosis among the population in both developed or developing countries is anticipated to rapidly increase the POCT market. However, there is no short path from the lab bench to commercialization for a POCT system. With the recent development of various plasmonics-enhanced diagnostic methods, more field testing and clinical validation studies will be needed to move the technology forward beyond the proof-of-concept laboratory demonstration. This requires a deep collaboration between research groups across the disciplines to perform well-designed field tests and pilot studies. For example, detection of biomarkers in spiked samples in the laboratory setting is totally different from detection of the same targets in complicated real sample matrix in the field. The stability and robustness of newly developed POCT will need to be thoroughly tested under different operational conditions (temperature, humidity, etc.). Therefore, extensive clinical validation and comparison studies are needed, which will constitute an important step to meet the requirement of the stringent government rules and regulations to be commercialized eventually.

\section{Conclusion and outlook}

In this review, we summarized the recent development of plasmonics-based biomolecular assays by surveying emerging applications for protein and nucleic acid biomarker detections. Utilizing the unique surfaceenhanced properties of plasmonic nanomaterials, including but not limited to SPR, plasmonic coupling, SERS, and SEF, these sensing strategies have shown great potential to be integrated with traditional, "gold standard" molecular diagnostic methods such as ELISA and DNA amplification to improve detection sensitivity, simplify procedure and readout mechanism, shorten assay time, and reduce sample volumes. Furthermore, integration of plasmonic molecular assays with portable platforms, such as microfluidics, wearable devices, and mobile phone readers has greatly accelerated the transformation of conventional benchtop assay protocols to become powerful POC tests for disease diagnosis. While several challenges still remain for POC implementation of plasmonic assays, we expect these systems to play an increasing important role in biomedicine and mobile health in the near future.

\section{Acknowledgements}

This work was supported by the Chancellor's Faculty Excellence Program and the Kenan Institute for 
Engineering, Technology \& Science (KIETS) at NC State.

\section{References}

[1] Gubala, V.; Harris, L. F.; Ricco, A. J.; Tan, M. X.; Williams, D. E. Point of care diagnostics: Status and future. Anal. Chem. 2012, 84, 487-515.

[2] In vitro diagnostics: Technologies and global markets, ReportLinker, 2017, https:/www.giiresearch.com/report/ bc345450-vitro-diagnostics-technologies-global-markets.ht $\mathrm{ml}$ (accessed May 7, 2018).

[3] Bauer, W. S.; Gulka, C. P.; Silva-Baucage, L.; Adams, N. M.; Haselton, F. R.; Wright, D. W. Metal affinity-enabled capture and release antibody reagents generate a multiplex biomarker enrichment system that improves detection limits of rapid diagnostic tests. Anal. Chem. 2017, 89, 10216-10223.

[4] Wei, T. Y.; Fu, Y.; Chang, K. H.; Lin, K. J.; Lu, Y. J.; Cheng, C. M. Point-of-care devices using disease biomarkers to diagnose neurodegenerative disorders. Trends Biotechnol. 2018, 36, 290-303.

[5] Tüdős, A. J.; Besselink, G. A. J.; Schasfoort, R. B. M. Trends in miniaturized total analysis systems for point-of-care testing in clinical chemistry. Lab Chip 2001, 1, 83-95.

[6] Duffy, D.; Mottez, E.; Ainsworth, S.; Buivan, T. P.; Baudin, A.; Vray, M.; Reed, B.; Fontanet, A.; Rohel, A.; PetrovSanchez, V. et al. An in vitro diagnostic certified point of care single nucleotide test for $I L 28 B$ polymorphisms. PLoS One 2017, 12, e0183084.

[7] Zarei, M. Portable biosensing devices for point-of-care diagnostics: Recent developments and applications. $\operatorname{Tr} A C$ Trends Anal. Chem. 2017, 91, 26-41.

[8] Wang, Y. Y.; Zhou, J. H.; Li, J. H. Construction of plasmonic nano-biosensor-based devices for point-of-care testing. Small Methods 2017, 1, 1700197.

[9] Silva, B. M. C.; Rodrigues, J. J. P. C.; de la Torre Díez, I.; López-Coronado, M.; Saleem, K. Mobile-health: A review of current state in 2015. J. Biomed. Inform. 2015, 56, 265-272.

[10] Laktabai, J.; Platt, A.; Menya, D.; Turner, E. L.; Aswa, D.; Kinoti, S.; O'Meara, W. P. A mobile health technology platform for quality assurance and quality improvement of malaria diagnosis by community health workers. PLoS One 2018, 13, e0191968.

[11] Howes, P. D.; Rana, S.; Stevens, M. M. Plasmonic nanomaterials for biodiagnostics. Chem. Soc. Rev. 2014, 43, 3835-3853.

[12] Zhou, W.; Gao, X.; Liu, D. B.; Chen, X. Y. Gold nanoparticles for in vitro diagnostics. Chem. Rev. 2015, 115, 10575-10636.
[13] Li, M.; Cushing, S. K.; Wu, N. Q. Plasmon-enhanced optical sensors: A review. Analyst 2015, 140, 386-406.

[14] Unser, S.; Bruzas, I.; He, J.; Sagle, L. Localized surface plasmon resonance biosensing: Current challenges and approaches. Sensors 2015, 15, 15684-15716.

[15] Sannomiya, T.; Vörös, J. Single plasmonic nanoparticles for biosensing. Trends Biotechnol. 2011, 29, 343-351.

[16] Tokel, O.; Inci, F.; Demirci, U. Advances in plasmonic technologies for point of care applications. Chem. Rev. 2014, 114, 5728-5752.

[17] Sun, J. S.; Xianyu, Y.; Jiang, X. Y. Point-of-care biochemical assays using gold nanoparticle-implemented microfluidics. Chem. Soc. Rev. 2014, 43, 6239-6253.

[18] Lim, W. Q.; Gao, Z. Q. Plasmonic nanoparticles in biomedicine. Nano Today 2016, 11, 168-188.

[19] Kravets, V. G.; Schedin, F.; Jalil, R.; Britnell, L.; Gorbachev, R. V.; Ansell, D.; Thackray, B.; Novoselov, K. S.; Geim, A. K.; Kabashin, A. V. et al. Singular phase nano-optics in plasmonic metamaterials for label-free singlemolecule detection. Nat. Mater. 2013, 12, 304-309.

[20] Zijlstra, P.; Paulo, P. M. R.; Orrit, M. Optical detection of single non-absorbing molecules using the surface plasmon resonance of a gold nanorod. Nat. Nanotechnol. 2012, 7, 379-382.

[21] Kelly, K. L.; Coronado, E.; Zhao, L. L.; Schatz, G. C. The optical properties of metal nanoparticles: The influence of size, shape, and dielectric environment. J. Phys. Chem. B 2003, 107, 668-677.

[22] Yang, X. J.; Yu, Y. B.; Gao, Z. Q. A highly sensitive plasmonic DNA assay based on triangular silver nanoprism etching. ACS Nano 2014, 8, 4902-4907.

[23] Fathi, F.; Rezabakhsh, A.; Rahbarghazi, R.; Rashidi, M. R. Early-stage detection of VE-cadherin during endothelial differentiation of human mesenchymal stem cells using SPR biosensor. Biosens. Bioelectron. 2017, 96, 358-366.

[24] Sendroiu, I. E.; Warner, M. E.; Corn, R. M. Fabrication of silica-coated gold nanorods functionalized with DNA for enhanced surface plasmon resonance imaging biosensing applications. Langmuir 2009, 25, 11282-11284.

[25] Shankaran, D. R.; Gobi, K. V.; Miura, N. Recent advancements in surface plasmon resonance immunosensors for detection of small molecules of biomedical, food and environmental interest. Sensor. Actuat. B: Chem. 2007, 121, 158-177.

[26] Homola, J. Surface plasmon resonance sensors for detection of chemical and biological species. Chem. Rev. 2008, 108, $462-493$.

[27] Hoa, X. D.; Kirk, A. G.; Tabrizian, M. Towards integrated and sensitive surface plasmon resonance biosensors: A review of recent progress. Biosens. Bioelectron. 2007, 23, 151-160. 
[28] Homola, J.; Yee, S. S.; Gauglitz, G. Surface plasmon resonance sensors: Review. Sensor. Actuat. B: Chem. 1999, 54, 3-15.

[29] Masson, J. F. Surface plasmon resonance clinical biosensors for medical diagnostics. ACS Sens. 2017, 2, 16-30.

[30] Zhao, W. A.; Brook, M. A.; Li, Y. F. Design of gold nanoparticle-based colorimetric biosensing assays. ChemBioChem 2008, 9, 2363-2371.

[31] Ngo, H. T.; Wang, H. N.; Fales, A. M.; Vo-Dinh, T. Label-free DNA biosensor based on SERS Molecular Sentinel on Nanowave chip. Anal. Chem. 2013, 85, 6378-6383.

[32] Bauch, M.; Toma, K.; Toma, M.; Zhang, Q. W.; Dostalek, J. Plasmon-enhanced fluorescence biosensors: A review. Plasmonics 2014, 9, 781-799.

[33] Willets, K. A.; Van Duyne, R. P. Localized surface plasmon resonance spectroscopy and sensing. Annu. Rev. Phys. Chem. 2007, 58, 267-297.

[34] Mayer, K. M.; Hafner, J. H. Localized surface plasmon resonance sensors. Chem. Rev. 2011, 111, 3828-3857.

[35] Chen, H. J.; Kou, X. S.; Yang, Z.; Ni, W. H.; Wang, J. F. Shape- and size-dependent refractive index sensitivity of gold nanoparticles. Langmuir 2008, 24, 5233-5237.

[36] Zhou, X.; Wong, T. I.; Song, H. Y.; Wu, L.; Wang, Y.; Bai, P.; Kim, D. H.; Ng, S. H.; Tse, M. S.; Knoll, W. Development of localized surface plasmon resonance-based point-of-care system. Plasmonics 2014, 9, 835-844.

[37] Inci, F.; Filippini, C.; Baday, M.; Ozen, M. O.; Calamak, S.; Durmus, N. G.; Wang, S. Q.; Hanhauser, E.; Hobbs, K. S.; Juillard, F. et al. Multitarget, quantitative nanoplasmonic electrical field-enhanced resonating device $\left(\mathrm{NE}^{2} \mathrm{RD}\right)$ for diagnostics. Proc. Natl. Acad. Sci. USA 2015, 112, E4354E4363.

[38] Tang, L. H.; Li, J. H. Plasmon-based colorimetric nanosensors for ultrasensitive molecular diagnostics. ACS Sens. 2017, 2, 857-875.

[39] Elghanian, R.; Storhoff, J. J.; Mucic, R. C.; Letsinger, R. L.; Mirkin, C. A. Selective colorimetric detection of polynucleotides based on the distance-dependent optical properties of gold nanoparticles. Science 1997, 277, 1078-1081.

[40] Cutler, J. I.; Auyeung, E.; Mirkin, C. A. Spherical nucleic acids. J. Am. Chem. Soc. 2012, 134, 1376-1391.

[41] Lee, J. S.; Han, M. S.; Mirkin, C. A. Colorimetric detection of mercuric ion $\left(\mathrm{Hg}^{2+}\right)$ in aqueous media using DNAfunctionalized gold nanoparticles. Angew. Chem., Int. Ed. 2007, 46, 4093-4096.

[42] Jia, Y. X.; Guo, Y. M.; Wang, S. W.; Chen, W. W.; Zhang, J. J.; Zheng, W. S.; Jiang, X. Y. Nanocrystalline cellulose mediated seed-growth for ultra-robust colorimetric detection of hydrogen sulfide. Nanoscale 2017, 9, 9811-9817.
[43] Gu, Y.; Song, J.; Li, M. X.; Zhang, T. T.; Zhao, W.; Xu, J. J.; Liu, M. L.; Chen, H. Y. Ultrasensitive microRNA assay via surface plasmon resonance responses of $\mathrm{Au} @ \mathrm{Ag}$ nanorods etching. Anal. Chem. 2017, 89, 10585-10591.

[44] Kim, J. Y.; Zeng, Z. C.; Xiao, L. F.; Schultz, Z. D. Elucidating protein/ligand recognition with combined surface plasmon resonance and surface enhanced Raman spectroscopy. Anal. Chem. 2017, 89, 13074-13081.

[45] Muehlethaler, C.; Leona, M.; Lombardi, J. R. Review of surface enhanced Raman scattering applications in forensic science. Anal. Chem. 2016, 88, 152-169.

[46] Lu, J. D.; Spasic, D.; Delport, F.; Van Stappen, T.; Detrez, I.; Daems, D.; Vermeire, S.; Gils, A.; Lammertyn, J. Immunoassay for detection of infliximab in whole blood using a fiber-optic surface plasmon resonance biosensor. Anal. Chem. 2017, 89, 3664-3671.

[47] Kim, S.; Wark, A. W.; Lee, H. J. Femtomolar detection of tau proteins in undiluted plasma using surface plasmon resonance. Anal. Chem. 2016, 88, 7793-7799.

[48] Kim, H.; Lee, J. U.; Song, S.; Kim, S.; Sim, S. J. A shape-code nanoplasmonic biosensor for multiplex detection of Alzheimer's disease biomarkers. Biosens. Bioelectron. 2018, 101, 96-102.

[49] Bui, M. P. N.; Ahmed, S.; Abbas, A. Single-digit pathogen and attomolar detection with the naked eye using liposomeamplified plasmonic immunoassay. Nano Lett. 2015, 15, 6239-6246.

[50] Valentini, P.; Pompa, P. P. A universal polymerase chain reaction developer. Angew. Chem., Int. Ed. 2016, 55, 2157-2160.

[51] Xiong, L. H.; He, X. W.; Xia, J. J.; Ma, H. W.; Yang, F.; Zhang, Q.; Huang, D.; Chen, L.; Wu, C. L.; Zhang, X. M. et al. Highly sensitive naked-eye assay for enterovirus 71 detection based on catalytic nanoparticle aggregation and immunomagnetic amplification. ACS Appl. Mater. Interfaces 2017, 9, 14691-14699.

[52] Teengam, P.; Siangproh, W.; Tuantranont, A.; Vilaivan, T.; Chailapakul, O.; Henry, C. S. Multiplex paper-based colorimetric DNA sensor using pyrrolidinyl peptide nucleic acid-induced AgNPs aggregation for detecting MERS-CoV, MTB, and HPV oligonucleotides. Anal. Chem. 2017, 89, 5428-5435.

[53] Chuong, T. T.; Pallaoro, A.; Chaves, C. A.; Li, Z.; Lee, J.; Eisenstein, M.; Stucky, G. D.; Moskovits, M.; Soh, H. T. Dual-reporter SERS-based biomolecular assay with reduced false-positive signals. Proc. Natl. Acad. Sci. USA 2017, 114, 9056-9061.

[54] Tang, B. C.; Wang, J. J.; Hutchison, J. A.; Ma, L.; Zhang, N.; Guo, H.; Hu, Z. B.; Li, M.; Zhao, Y. L. Ultrasensitive, 
multiplex Raman frequency shift immunoassay of liver cancer biomarkers in physiological media. ACS Nano 2016, 10, 871-879.

[55] Fu, X. L.; Cheng, Z. Y.; Yu, J. M.; Choo, P.; Chen, L. X.; Choo, J. A SERS-based lateral flow assay biosensor for highly sensitive detection of HIV-1 DNA. Biosens. Bioelectron. 2016, 78, 530-537.

[56] Mei, Z.; Tang, L. Surface-plasmon-coupled fluorescence enhancement based on ordered gold nanorod array biochip for ultrasensitive DNA analysis. Anal. Chem. 2017, 89, 633-639.

[57] Zhang, B.; Jarrell, J. A.; Price, J. V.; Tabakman, S. M.; Li, Y. G.; Gong, M.; Hong, G. S.; Feng, J.; Utz, P. J.; Dai, H. J. An integrated peptide-antigen microarray on plasmonic gold films for sensitive human antibody profiling. PLoS One 2013, 8, e71043.

[58] Liu, B.; Li, Y. L.; Wan, H.; Wang, L.; Xu, W.; Zhu, S. J.; Liang, Y. Y.; Zhang, B.; Lou, J. T.; Dai, H. J. et al. High performance, multiplexed lung cancer biomarker detection on a plasmonic gold chip. Adv. Funct. Mater. 2016, 26, 7994-8002.

[59] Song, H. Y.; Wong, T. I.; Sadovoy, A.; Wu, L.; Bai, P.; Deng, J.; Guo, S. F.; Wang, Y.; Knoll, W.; Zhou, X. D. Imprinted gold 2D nanoarray for highly sensitive and convenient PSA detection via plasmon excited quantum dots. Lab Chip 2015, 15, 253-263.

[60] Tu, X. Y.; Muhammad, P.; Liu, J.; Ma, Y. Y.; Wang, S. S.; Yin, D. Y.; Liu, Z. Molecularly imprinted polymer-based plasmonic immunosandwich assay for fast and ultrasensitive determination of trace glycoproteins in complex samples. Anal. Chem. 2016, 88, 12363-12370.

[61] Liu, K.; Bai, Y. C.; Zhang, L.; Yang, Z. B.; Fan, Q. K.; Zheng, H. Q.; Yin, Y. D.; Gao, C. B. Porous Au-Ag nanospheres with high-density and highly accessible hotspots for SERS analysis. Nano Lett. 2016, 16, 3675-3681.

[62] Zheng, J. W.; Zhou, Y. G.; Li, X. W.; Ji, Y.; Lu, T. H.; Gu, R. A. Surface-enhanced Raman scattering of 4-aminothiophenol in assemblies of nanosized particles and the macroscopic surface of silver. Langmuir 2003, 19, 632-636.

[63] Yang, K. H.; Liu, Y. C.; Yu, C. C. Enhancements in intensity and stability of surface-enhanced Raman scattering on optimally electrochemically roughened silver substrates. J. Mater. Chem. 2008, 18, 4849-4855.

[64] Chang, C. C.; Hsu, T. C.; Liu, Y. C.; Yang, K. H. Surface-enhanced Raman scattering-active silver substrates electrochemically prepared in solutions containing bielectrolytes. J. Mater. Chem. 2011, 21, 6660-6667.

[65] Shin, H. H.; Yeon, G. J.; Choi, H.-K.; Park, S. M.; Lee, K. S.; Kim, Z. H. Frequency-domain proof of the existence of atomic-scale SERS hot-spots. Nano Lett. 2018, 18, 262-271.

[66] Qian, X. M.; Peng, X. H.; Ansari, D. O.; Yin-Goen, Q.; Chen, G. Z.; Shin, D. M.; Yang, L.; Young, A. N.; Wang, M. D.; Nie, S. M. In vivo tumor targeting and spectroscopic detection with surface-enhanced Raman nanoparticle tags. Nat. Biotechnol. 2008, 26, 83-90.

[67] Marks, H.; Schechinger, M.; Garza, J.; Locke, A.; Coté, G. Surface enhanced Raman spectroscopy (SERS) for in vitro diagnostic testing at the point of care. Nanophotonics 2017, 6, 681-701.

[68] Granger, J. H.; Schlotter, N. E.; Crawford, A. C.; Porter, M. D. Prospects for point-of-care pathogen diagnostics using surface-enhanced Raman scattering (SERS). Chem. Soc. Rev. 2016, 45, 3865-3882

[69] Vasilev, K.; Knoll, W.; Kreiter, M. Fluorescence intensities of chromophores in front of a thin metal film. J. Chem. Phys. 2004, 120, 3439-3445.

[70] Enderlein, J. A theoretical investigation of single-molecule fluorescence detection on thin metallic layers. Biophys. $J$. 2000, 78, 2151-2158.

[71] Loebermann, T.; Knoll, W. Surface-plasmon field-enhanced fluorescence spectroscopy. Colloids Surf. A: Physicochem. Eng. Aspects 2000, 171, 115-130.

[72] Acuna, G. P.; Möller, F. M.; Holzmeister, P.; Beater, S.; Lalkens, B.; Tinnefeld, P. Fluorescence enhancement at docking sites of DNA-directed self-assembled nanoantennas. Science 2012, 338, 506-510.

[73] Kinkhabwala, A.; Yu, Z. F.; Fan, S. H.; Avlasevich, Y.; Müllen, K.; Moerner, W. E. Large single-molecule fluorescence enhancements produced by a bowtie nanoantenna. Nat. Photonics 2009, 3, 654-657.

[74] Punj, D.; Mivelle, M.; Moparthi, S. B.; van Zanten, T. S.; Rigneault, H.; van Hulst, N. F.; García-Parajó, M. F.; Wenger, J. A plasmonic 'antenna-in-box' platform for enhanced single-molecule analysis at micromolar concentrations. Nat. Nanotechnol. 2013, 8, 512-516.

[75] Zhang, B.; Price, J.; Hong, G. S.; Tabakman, S. M.; Wang, H. L.; Jarrell, J. A.; Feng, J.; Utz, P. J.; Dai, H. J. Multiplexed cytokine detection on plasmonic gold substrates with enhanced near-infrared fluorescence. Nano Res. 2013, 6, 113-120.

[76] Tabakman, S. M.; Lau, L.; Robinson, J. T.; Price, J.; Sherlock, S. P.; Wang, H. L.; Zhang, B.; Chen, Z.; Tangsombatvisit, S.; Jarrell, J. A. et al. Plasmonic substrates for multiplexed protein microarrays with femtomolar sensitivity and broad dynamic range. Nat. Commun. 2011, 2, 466.

[77] Zhang, B.; Kumar, R. B.; Dai, H. J.; Feldman, B. J. A plasmonic chip for biomarker discovery and diagnosis of type 1 diabetes. Nat. Med. 2014, 20, 948-953. 
[78] Hong, G. S.; Tabakman, S. M.; Welsher, K.; Chen, Z.; Robinson, J. T.; Wang, H. L.; Zhang, B.; Dai, H. J. Nearinfrared-fluorescence-enhanced molecular imaging of live cells on gold substrates. Angew. Chem., Int. Ed. 2011, 50, 4644-4648.

[79] Guo, L. H.; Jackman, J. A.; Yang, H. H.; Chen, P.; Cho, N. J.; Kim, D. H. Strategies for enhancing the sensitivity of plasmonic nanosensors. Nano Today 2015, 10, 213-239.

[80] Engvall, E.; Perlmann, P. Enzyme-linked immunosorbent assay (ELISA) quantitative assay of immunoglobulin G. Immunochemistry 1971, 8, 871-874.

[81] Liang, Y.; Huang, X. L.; Chen, X. R.; Zhang, W. J.; Ping, G.; Xiong, Y. H. Plasmonic ELISA for naked-eye detection of ochratoxin A based on the tyramine- $\mathrm{H}_{2} \mathrm{O}_{2}$ amplification system. Sensor. Actuat. B: Chem. 2018, 259, 162-169.

[82] Zhang, S. Y.; Garcia-D’Angeli, A.; Brennan, J. P.; Huo, Q. Predicting detection limits of enzyme-linked immunosorbent assay (ELISA) and bioanalytical techniques in general. Analyst 2014, 139, 439-445.

[83] de la Rica, R.; Stevens, M. M. Plasmonic ELISA for the ultrasensitive detection of disease biomarkers with the naked eye. Nat. Nanotechnol. 2012, 7, 821-824.

[84] de la Rica, R.; Stevens, M. M. Plasmonic ELISA for the detection of analytes at ultralow concentrations with the naked eye. Nat. Protoc. 2013, 8, 1759-1764.

[85] Cecchin, D.; de la Rica, R.; Bain, R. E. S.; Finnis, M. W.; Stevens, M. M.; Battaglia, G. Plasmonic ELISA for the detection of gp120 at ultralow concentrations with the naked eye. Nanoscale 2014, 6, 9559-9562.

[86] Peng, M. P.; Ma, W.; Long, Y. T. Alcohol Dehydrogenasecatalyzed gold nanoparticle seed-mediated growth allows reliable detection of disease biomarkers with the naked eye. Anal. Chem. 2015, 87, 5891-5896.

[87] Ambrosi, A.; Airò, F.; Merkoçi, A. Enhanced gold nanoparticle based ELISA for a breast cancer biomarker. Anal. Chem. 2010, 82, 1151-1156.

[88] Liu, D. B.; Yang, J.; Wang, H. F.; Wang, Z. L.; Huang, X. L.; Wang, Z. T.; Niu, G.; Hight Walker, A. R.; Chen, X. Y. Glucose oxidase-catalyzed growth of gold nanoparticles enables quantitative detection of attomolar cancer biomarkers. Anal. Chem. 2014, 86, 5800-5806.

[89] Zhou, F.; Wang, M. M.; Yuan, L.; Cheng, Z. P.; Wu, Z. Q.; Chen, H. Sensitive sandwich ELISA based on a gold nanoparticle layer for cancer detection. Analyst 2012, 137, 1779-1784.

[90] Jia, C. P.; Zhong, X. Q.; Hua, B.; Liu, M. Y.; Jing, F. X.; Lou, X. H.; Yao, S. H.; Xiang, J. Q.; Jin, Q. H.; Zhao, J. L. Nano-ELISA for highly sensitive protein detection. Biosens. Bioelectron. 2009, 24, 2836-2841.
[91] Liu, M. Y.; Jia, C. P.; Huang, Y. Y.; Lou, X. H.; Yao, S. H.; Jin, Q. H.; Zhao, J. L.; Xiang, J. Q. Highly sensitive protein detection using enzyme-labeled gold nanoparticle probes. Analyst 2010, 135, 327-331.

[92] Liang, J. J.; Yao, C. Z.; Li, X. Q.; Wu, Z.; Huang, C. H.; Fu, Q. Q.; Lan, C. F.; Cao, D. L.; Tang, Y. Silver nanoprism etching-based plasmonic ELISA for the high sensitive detection of prostate-specific antigen. Biosens. Bioelectron. 2015, 69, 128-134.

[93] Nie, X. M.; Huang, R.; Dong, C. X.; Tang, L. J.; Gui, R.; Jiang, J. H. Plasmonic ELISA for the ultrasensitive detection of Treponema pallidum. Biosens. Bioelectron. 2014, 58, 314-319.

[94] Rissin, D. M.; Kan, C. W.; Campbell, T. G.; Howes, S. C.; Fournier, D. R.; Song, L.; Piech, T.; Patel, P. P.; Chang, L.; Rivnak, A. J. et al. Single-molecule enzyme-linked immunosorbent assay detects serum proteins at subfemtomolar concentrations. Nat. Biotechnol. 2010, 28, 595-599.

[95] Chen, S.; Svedendahl, M.; Antosiewicz, T. J.; Käll, M. Plasmon-enhanced enzyme-linked immunosorbent assay on large arrays of individual particles made by electron beam lithography. ACS Nano 2013, 7, 8824-8832.

[96] Chen, S.; Svedendahl, M.; van Duyne, R. P.; Käll, M. Plasmon-enhanced colorimetric ELISA with single molecule sensitivity. Nano Lett. 2011, 11, 1826-1830.

[97] Sia, S. K.; Linder, V.; Parviz, B. A.; Siegel, A.; Whitesides, G. M. An integrated approach to a portable and low-cost immunoassay for resource-poor settings. Angew. Chem., Int. Ed. 2004, 43, 498-502.

[98] Gupta, S.; Huda, S.; Kilpatrick, P. K.; Velev, O. D. Characterization and optimization of gold nanoparticlebased silver-enhanced immunoassays. Anal. Chem. 2007, 79, 3810-3820.

[99] Yang, C. T.; Wu, L.; Bai, P.; Thierry, B. Investigation of plasmonic signal enhancement based on long range surface plasmon resonance with gold nanoparticle tags. J. Mater. Chem. C 2016, 4, 9897-9904.

[100] Lyon, L. A.; Musick, M. D.; Natan, M. J. Colloidal Au-enhanced surface plasmon resonance immunosensing. Anal. Chem. 1998, 70, 5177-5183.

[101] Kwon, M. J.; Lee, J.; Wark, A. W.; Lee, H. J. Nanoparticleenhanced surface plasmon resonance detection of proteins at attomolar concentrations: Comparing different nanoparticle shapes and sizes. Anal. Chem. 2012, 84, 1702-1707.

[102] Guarrotxena, N.; Liu, B.; Fabris, L.; Bazan, G. C. Antitags: Nanostructured tools for developing SERS-based ELISA analogs. Adv. Mater. 2010, 22, 4954-4958.

[103] Li, X. Y.; Kuznetsova, T.; Cauwenberghs, N.; Wheeler, M.; Maecker, H.; Wu, J. C.; Haddad, F.; Dai, H. J. Autoantibody 
profiling on a plasmonic nano-gold chip for the early detection of hypertensive heart disease. Proc. Natl. Acad. Sci. USA 2017, 114, 7089-7094.

[104] Zhao, Y. X.; Chen, F.; Li, Q.; Wang, L. H.; Fan, C. H. Isothermal amplification of nucleic acids. Chem. Rev. 2015, 115, 12491-12545.

[105] White, P. L.; Hibbitts, S. J.; Perry, M. D.; Green, J.; Stirling, E.; Woodford, L.; McNay, G.; Stevenson, R.; Barnes, R. A. Evaluation of a commercially developed semiautomated PCR-surface-enhanced Raman scattering assay for diagnosis of invasive fungal disease. J. Clin. Microbiol. 2014, 52, 3536-3543.

[106] Almassian, D. R.; Cockrell, L. M.; Nelson, W. M. Portable nucleic acid thermocyclers. Chem. Soc. Rev. 2013, 42, 8769-8798.

[107] Zhang, C. S.; Xing, D. Single-molecule DNA amplification and analysis using microfluidics. Chem. Rev. 2010, 110, 4910-4947.

[108] Lee, D.; Chou, W. P.; Yeh, S. H.; Chen, P. J.; Chen, P. H. DNA detection using commercial mobile phones. Biosens. Bioelectron. 2011, 26, 4349-4354.

[109] Myers, F. B.; Henrikson, R. H.; Bone, J.; Lee, L. P. A handheld point-of-care genomic diagnostic system. PLoS One 2013, 8, e70266.

[110] Priye, A.; Wong, S.; Bi, Y. P.; Carpio, M.; Chang, J.; Coen, M.; Cope, D.; Harris, J.; Johnson, J.; Keller, A. et al. Lab-on-a-Drone: Toward pinpoint deployment of smartphone-enabled nucleic acid-based diagnostics for mobile health care. Anal. Chem. 2016, 88, 4651-4660.

[111] Sawata, S.; Kai, E.; Ikebukuro, K.; Iida, T.; Honda, T.; Karube, I. Application of peptide nucleic acid to the direct detection of deoxyribonucleic acid amplified by polymerase chain reaction. Biosens. Bioelectron. 1999, 14, 397-404.

[112] Kai, E.; Sawata, S.; Ikebukuro, K.; Iida, T.; Honda, T.; Karube, I. Detection of PCR products in solution using surface plasmon resonance. Anal. Chem. 1999, 71, 796-800.

[113] Wu, J. L.; Huang, Y.; Bian, X. T.; Li, D. D.; Cheng, Q.; Ding, S. J. Biosensing of BCR/ABL fusion gene using an intensity-interrogation surface plasmon resonance imaging system. Opt. Commun. 2016, 377, 24-32.

[114] Yao, D.; Yu, F.; Kim, J.; Scholz, J.; Nielsen, P. E.; Sinner, E. K.; Knoll, W. Surface plasmon field-enhanced fluorescence spectroscopy in PCR product analysis by peptide nucleic acid probes. Nucleic Acids Res. 2004, 32, e177.

[115] Faulds, K.; Barbagallo, R. P.; Keer, J. T.; Smith, W. E.; Graham, D. SERRS as a more sensitive technique for the detection of labelled oligonucleotides compared to fluorescence. Analyst 2004, 129, 567-568.

[116] Van Lierop, D.; Larmour, I. A.; Faulds, K.; Graham, D.
SERS primers and their mode of action for pathogen DNA detection. Anal. Chem. 2013, 85, 1408-1414.

[117] Cai, M.; Li, F.; Zhang, Y.; Wang, Q. B. One-pot polymerase chain reaction with gold nanoparticles for rapid and ultrasensitive DNA detection. Nano Res. 2010, 3, 557-563.

[118] Deng, H.; Xu, Y.; Liu, Y. H.; Che, Z. J.; Guo, H. L.; Shan, S. X.; Sun, Y.; Liu, X. F.; Huang, K. Y.; Ma, X. W. et al. Gold nanoparticles with asymmetric polymerase chain reaction for colorimetric detection of DNA sequence. Anal. Chem. 2012, 84, 1253-1258.

[119] Li, F.; Li, F. L.; Yang, G. T.; Aguilar, Z. P.; Lai, W. H.; $\mathrm{Xu}, \mathrm{H}$. Y. Asymmetric polymerase chain assay combined with propidium monoazide treatment and unmodified gold nanoparticles for colorimetric detection of viable emetic Bacillus cereus in milk. Sensor. Actuat. B: Chem. 2018, 255, 1455-1461.

[120] Roche, P. J. R.; Beitel, L. K.; Khan, R.; Lumbroso, R.; Najih, M.; Cheung, M. C. K.; Thiemann, J.; Veerasubramanian, V.; Trifiro, M.; Chodavarapu, V. P. et al. Demonstration of a plasmonic thermocycler for the amplification of human androgen receptor DNA. Analyst 2012, 137, 4475-4481.

[121] Son, J. H.; Cho, B.; Hong, S.; Lee, S. H.; Hoxha, O.; Haack, A. J.; Lee, L. P. Ultrafast photonic PCR. Light-Sci. Appl. 2015, 4, e280.

[122] Lee, J. H.; Cheglakov, Z.; Yi, J.; Cronin, T. M.; Gibson, K. J.; Tian, B. Z.; Weizmann, Y. Plasmonic photothermal gold bipyramid nanoreactors for ultrafast real-time bioassays. J. Am. Chem. Soc. 2017, 139, 8054-8057.

[123] Roche, P. J. R.; Najih, M.; Lee, S. S.; Beitel, L. K.; Carnevale, M. L.; Paliouras, M.; Kirk, A. G.; Trifiro, M. A. Real time plasmonic qPCR: How fast is ultra-fast? 30 cycles in 54 seconds. Analyst 2017, 142, 1746-1755.

[124] Yu, T.; Dai, P. P.; Xu, J. J.; Chen, H. Y. Highly sensitive colorimetric cancer cell detection based on dual signal amplification. ACS Appl. Mater. Interfaces 2016, 8, 44344441.

[125] Zhang, X. X.; Xiao, K. Y.; Cheng, L. W.; Chen, H.; Liu, B. H.; Zhang, S.; Kong, J. L. Visual and highly sensitive detection of cancer cells by a colorimetric aptasensor based on cell-triggered cyclic enzymatic signal amplification. Anal. Chem. 2014, 86, 5567-5572.

[126] Xu, W.; Xue X. J.; Li, T. H.; Zeng H. Q.; Liu X. G. Ultrasensitive and selective colorimetric DNA detection by nicking endonuclease assisted nanoparticle amplification. Angew. Chem., Int. Ed. 2009, 48, 6849-6852.

[127] Wong, J. K.; Yip, S. P.; Lee, T. M. H. Ultrasensitive and closed-tube colorimetric loop-mediated isothermal amplification assay using carboxyl-modified gold nanoparticles. Small 2014, 10, 1495-1499. 
[128] Bozorgmehr, A.; Yazdanparast, R.; Mollasalehi, H. Noncrosslinking gold nanoprobe-LAMP for simple, colorimetric, and specific detection of Salmonella typhi. J. Nanopart. Res. 2016, 18, 351.

[129] Qin, A. L.; Fu, L. T.; Wong, J. K. F.; Chau, L. Y.; Yip, S. P.; Lee, T. M. H. Precipitation of PEG/carboxyl-modified gold nanoparticles with magnesium pyrophosphate: A new platform for real-time monitoring of loop-mediated isothermal amplification. ACS Appl. Mater. Interfaces 2017, 9, 10472-10480.

[130] Li, J. S.; Deng, T.; Chu, X.; Yang, R. H.; Jiang, J. H.; Shen, G. L.; Yu, R. Q. Rolling circle amplification combined with gold nanoparticle aggregates for highly sensitive identification of single-nucleotide polymorphisms. Anal. Chem. 2010, 82, 2811-2816.

[131] Liu, P.; Yang, X. H.; Sun, S.; Wang, Q.; Wang, K. M.; Huang, J.; Liu, J. B.; He, L. L. Enzyme-free colorimetric detection of DNA by using gold nanoparticles and hybridization chain reaction amplification. Anal. Chem. 2013, 85, 7689-7695.

[132] Rana, M.; Balcioglu, M.; Kovach, M.; Hizir, M. S.; Robertson, N. M.; Khan, I.; Yigit, M. V. Reprogrammable multiplexed detection of circulating oncomiRs using hybridization chain reaction. Chem. Commun. 2016, 52, 3524-3527.

[133] Yun, W.; Jiang, J. L.; Cai, D. Z.; Zhao, P. X.; Liao, J. S.; Sang, G. Ultrasensitive visual detection of DNA with tunable dynamic range by using unmodified gold nanoparticles and target catalyzed hairpin assembly amplification. Biosens. Bioelectron. 2016, 77, 421-427.

[134] Kato, D.; Oishi, M. Ultrasensitive detection of DNA and RNA based on enzyme-free click chemical ligation chain reaction on dispersed gold nanoparticles. ACS Nano 2014, 8, 9988-9997.

[135] Oishi, M.; Sugiyama, S. An efficient particle-based DNA circuit system: Catalytic disassembly of DNA/PEG-modified gold nanoparticle-magnetic bead composites for colorimetric detection of miRNA. Small 2016, 12, 5153-5158.

[136] Nawattanapaiboon, K.; Kiatpathomchai, W.; Santanirand, P.; Vongsakulyanon, A.; Amarit, R.; Somboonkaew, A.; Sutapun, B.; Srikhirin, T. SPR-DNA array for detection of methicillin-resistant Staphylococcus aureus (MRSA) in combination with loop-mediated isothermal amplification. Biosens. Bioelectron. 2015, 74, 335-340.

[137] He, P.; Qiao, W. P.; Liu, L. J.; Zhang, S. S. A highly sensitive surface plasmon resonance sensor for the detection of DNA and cancer cells by a target-triggered multiple signal amplification strategy. Chem. Commun. 2014, 50, 10718-10721.
[138] Zeng, K.; Li, H. Y.; Peng, Y. Y. Gold nanoparticle enhanced surface plasmon resonance imaging of microRNA-155 using a functional nucleic acid-based amplification machine. Microchim. Acta 2017, 184, 2637-2644.

[139] Sendroiu, I. E.; Gifford, L. K.; Lupták, A.; Corn, R. M. Ultrasensitive DNA microarray biosensing via in situ RNA transcription-based amplification and nanoparticle-enhanced SPR imaging. J. Am. Chem. Soc. 2011, 133, 4271-4273.

[140] Ding, X. J.; Cheng, W.; Li, Y. J.; Wu, J. L.; Li, X. M.; Cheng, Q.; Ding, S. J. An enzyme-free surface plasmon resonance biosensing strategy for detection of DNA and small molecule based on nonlinear hybridization chain reaction. Biosens. Bioelectron. 2017, 87, 345-351.

[141] Ding, X. J.; Yan, Y. R.; Li, S. Q.; Zhang, Y.; Cheng, W.; Cheng, Q.; Ding, S. J. Surface plasmon resonance biosensor for highly sensitive detection of microRNA based on DNA super-sandwich assemblies and streptavidin signal amplification. Anal. Chim. Acta 2015, 874, 59-65.

[142] Guo, B.; Cheng, W.; Xu, Y. J.; Zhou, X. Y.; Li, X. M.; Ding, X. J.; Ding, S. J. A simple surface plasmon resonance biosensor for detection of PML/RAR $\alpha$ based on heterogeneous fusion gene-triggered nonlinear hybridization chain reaction. Sci. Rep. 2017, 7, 14037.

[143] Yao, G. H.; Liang, R. P.; Yu, X. D.; Huang, C. F.; Zhang, L.; Qiu, J. D. Target-triggering multiple-cycle amplification strategy for ultrasensitive detection of adenosine based on surface plasma resonance techniques. Anal. Chem. 2015, 87, 929-936.

[144] Yao, G. H.; Liang, R. P.; Huang, C. F.; Zhang, L.; Qiu, J. D. Enzyme-free surface plasmon resonance aptasensor for amplified detection of adenosine via target-triggering strand displacement cycle and $\mathrm{Au}$ nanoparticles. Anal. Chim. Acta 2015, 871, 28-34.

[145] Wang, Q.; Liu, R. J.; Yang, X. H.; Wang, K. M.; Zhu, J. Q.; He, L. L.; Li, Q. Surface plasmon resonance biosensor for enzyme-free amplified microRNA detection based on gold nanoparticles and DNA supersandwich. Sensor. Actuat. B: Chem. 2016, 223, 613-620.

[146] Diao, W.; Tang, M.; Ding, S. J.; Li, X. M.; Cheng, W. B.; Mo, F.; Yan, X. Y.; Ma, H. M.; Yan, Y. R. Highly sensitive surface plasmon resonance biosensor for the detection of HIV-related DNA based on dynamic and structural DNA nanodevices. Biosens. Bioelectron. 2018, 100, 228-234.

[147] Yin, F. F.; Liu, H. Q.; Li, Q.; Gao, X.; Yin, Y. M.; Liu, D. B. Trace microRNA quantification by means of plasmonenhanced hybridization chain reaction. Anal. Chem. 2016, $88,4600-4604$.

[148] Hu, J.; Zhang, C. Y. Sensitive detection of nucleic acids with rolling circle amplification and surface-enhanced Raman 
scattering spectroscopy. Anal. Chem. 2010, 82, 8991-8997.

[149] Li, Y.; Lei, C. C.; Zeng, Y.; Ji, X. T.; Zhang, S. S. Sensitive SERS detection of DNA and lysozyme based on polymerase assisted cross strand-displacement amplification. Chem. Commun. 2012, 48, 10892-10894.

[150] Wang, S. Y.; Yang, H. W.; Zhang, H. T.; Yang, F. H.; Zhou, M. S.; Jia, C. W.; Lan, Y. L.; Ma, Y. M.; Zhou, L. Y.; Tian, S. et al. A surface plasmon resonance-based system to genotype human papillomavirus. Cancer Genet. Cytogenet. 2010, 200, 100-105.

[151] Qu, S.; Huang, J.; Zhao, J.; Zhao, X.; Deng, H.; Yang, H.; Chen, W.; Liu, L.; Zhang, L.; Gao, S. A comparison of matrix-assisted laser desorption/ionization time-of-flight mass spectrometry and surface plasmon resonance for genotyping of high-risk human papillomaviruses. Intervirology 2011, 54, 326-332.

[152] Okumura, S.; Kuroda, R.; Inouye, K. Single nucleotide polymorphism typing with a surface plasmon resonancebased sensor using hybridization enhancement blockers. Appl. Biochem. Biotechnol. 2014, 174, 494-505.

[153] Mariani, S.; Scarano, S.; Carrai, M.; Barale, R.; Minunni, M. Direct genotyping of C3435T single nucleotide polymorphism in unamplified human MDR1 gene using a surface plasmon resonance imaging DNA sensor. Anal. Bioanal. Chem. 2015, 407, 4023-4028.

[154] Zhong, X. B.; Reynolds, R.; Kidd, J. R.; Kidd, K. K.; Jenison, R.; Marlar, R. A.; Ward, D. C. Single-nucleotide polymorphism genotyping on optical thin-film biosensor chips. Proc. Natl. Acad. Sci. USA 2003, 100, 11559-11564.

[155] Li, Y.; Wark, A. W.; Lee, H. J.; Corn, R. M. Singlenucleotide polymorphism genotyping by nanoparticleenhanced surface plasmon resonance imaging measurements of surface ligation reactions. Anal. Chem. 2006, 78, 3158-3164.

[156] Hibbitts, S.; White, P. L.; Green, J.; McNay, G.; Graham, D.; Stevenson, R. Human papilloma virus genotyping by surface-enhanced Raman scattering. Anal. Methods 2014, 6, 1288-1290.

[157] Lowe, A. J.; Huh, Y. S.; Strickland, A. D.; Erickson, D.; Batt, C. A. Multiplex single nucleotide polymorphism genotyping utilizing ligase detection reaction coupled surface enhanced Raman spectroscopy. Anal. Chem. 2010, $82,5810-5814$.

[158] Shendure, J.; Ji, H. Next-generation DNA sequencing. Nat. Biotechnol. 2008, 26, 1135-1145.

[159] Metzker, M. L. Sequencing technologies - the next generation. Nat. Rev. Genet. 2010, 11, 31-46.

[160] Mardis, E. R. DNA sequencing technologies: 2006-2016. Nat. Protoc. 2017, 12, 213-218.
[161] Goodwin, S.; McPherson, J. D.; McCombie, W. R. Coming of age: Ten years of next-generation sequencing technologies. Nat. Rev. Genet. 2016, 17, 333-351.

[162] Rothberg, J. M.; Hinz, W.; Rearick, T. M.; Schultz, J.; Mileski, W.; Davey, M.; Leamon, J. H.; Johnson, K.; Milgrew, M. J.; Edwards, M. et al. An integrated semiconductor device enabling non-optical genome sequencing. Nature 2011, 475, 348-352.

[163] Ashton, P. M.; Nair, S.; Dallman, T.; Rubino, S.; Rabsch, W.; Mwaigwisya, S.; Wain, J.; O’Grady, J. MinION nanopore sequencing identifies the position and structure of a bacterial antibiotic resistance island. Nat. Biotechnol. 2015, 33, 296-300

[164] Eif, J.; Fehr, A.; Gray, J.; Luong, K.; Lyle, J.; Otto, G.; Peluso, P.; Rank, D.; Baybayan, P.; Bettman, B. et al. Real-time DNA sequencing from single polymerase molecules. Science 2009, 323, 133-138.

[165] Flusberg, B. A.; Webster, D. R.; Lee, J. H.; Travers, K. J.; Olivares, E. C.; Clark, T. A.; Korlach, J.; Turner, S. W. Direct detection of DNA methylation during single-molecule, real-time sequencing. Nat. Methods 2010, 7, 461-465.

[166] Levene, M. J.; Korlach, J.; Turner, S. W.; Foquet, M.; Craighead, H. G.; Webb, W. W. Zero-mode waveguides for single-molecule analysis at high concentrations. Science 2003, 299, 682-686.

[167] Bailo, E.; Deckert, V. Tip-enhanced Raman spectroscopy of single RNA strands: Towards a novel direct-sequencing method. Angew. Chem., Int. Ed. 2008, 47, 1658-1661.

[168] Palla, M.; Guo, W. J.; Shi, S. D.; Li, Z. M.; Wu, J.; Jockusch, S.; Guo, C.; Russo, J. J.; Turro, N. J.; Ju, J. Y. DNA sequencing by synthesis using 3'-O-azidomethyl nucleotide reversible terminators and surface-enhanced Raman spectroscopic detection. RSC Adv. 2014, 4, 4934249346.

[169] Fotouhi, B.; Ahmadi, V.; Faramarzi, V. Nano-plasmonicbased structures for DNA sequencing. Opt. Lett. 2016, 41, 4229-4232.

[170] Schmieder, S.; Weißpflog, J.; Danz, N.; Hübner, M.; Kreth, S.; Klotzbach, U.; Sonntag, F. Ultrasensitive SPR detection of miRNA-93 using antibody-enhanced and enzymatic signal amplification. Eng. Life Sci. 2017, 17, 1264-1270.

[171] Tokel, O.; Yildiz, U. H.; Inci, F.; Durmus, N. G.; Ekiz, O. O.; Turker, B.; Cetin, C.; Rao, S.; Sridhar, K.; Natarajan, N. et al. Portable microfluidic integrated plasmonic platform for pathogen detection. Sci. Rep. 2015, 5, 9152.

[172] Aćimović, S. S.; Ortega, M. A.; Sanz, V.; Berthelot, J.; Garcia-Cordero, J. L.; Renger, J.; Maerkl, S. J.; Kreuzer, M. P.; Quidant, R. LSPR chip for parallel, rapid, and 
sensitive detection of cancer markers in serum. Nano Lett. 2014, 14, 2636-2641.

[173] Wang, C.; Yu, C. X. Analytical characterization using surface-enhanced Raman scattering (SERS) and microfluidic sampling. Nanotechnology 2015, 26, 092001.

[174] Choi, N.; Lee, J.; Ko, J.; Jeon, J. H.; Rhie, G. E.; deMello, A. J.; Choo, J. Integrated SERS-based microdroplet platform for the automated immunoassay of F1 antigens in Yersinia pestis. Anal. Chem. 2017, 89, 8413-8420.

[175] Kim, D. J.; Jeon, T. Y.; Park, S. G.; Han, H. J.; Im, S. H.; Kim, D. H.; Kim, S. H. Uniform microgels containing agglomerates of silver nanocubes for molecular sizeselectivity and high SERS activity. Small 2017, 13, 1604048.

[176] Kurita, R.; Yanagisawa, H.; Yoshioka, K.; Niwa, O. On-chip sequence-specific immunochemical epigenomic analysis utilizing outward-turned cytosine in a DNA bulge with handheld surface plasmon resonance equipment. Anal. Chem. 2015, 87, 11581-11586.

[177] Wang, H. S.; Wang, C.; He, Y. K.; Xiao, F. N.; Bao, W. J.; Xia, X. H.; Zhou, G. J. Core-shell Ag@ $\mathrm{SiO}_{2}$ nanoparticles concentrated on a micro/nanofluidic device for surface plasmon resonance-enhanced fluorescent detection of highly reactive oxygen species. Anal. Chem. 2014, 86, 3013-3019.

[178] Li, J.; Skeete, Z.; Shan, S. Y.; Yan, S.; Kurzatkowska, K.; Zhao, W.; Ngo, Q. M.; Holubovska, P.; Luo, J.; Hepel, M. et al. Surface enhanced Raman scattering detection of cancer biomarkers with bifunctional nanocomposite probes. Anal. Chem. 2015, 87, 10698-10702.

[179] Yamada, K.; Shibata, H.; Suzuki, K.; Citterio, D. Toward practical application of paper-based microfluidics for medical diagnostics: State-of-the-art and challenges. Lab Chip 2017, 17, 1206-1249.

[180] Akyazi, T.; Basabe-Desmonts, L.; Benito-Lopez, F. Review on microfluidic paper-based analytical devices towards commercialisation. Anal. Chim. Acta 2018, 1001, 1-17.

[181] Yetisen, A. K.; Akram, M. S.; Lowe, C. R. Paper-based microfluidic point-of-care diagnostic devices. Lab Chip 2013, 13, 2210-2251.

[182] Yen, C. W.; de Puig, H.; Tam, J. O.; Gómez-Márquez, J.; Bosch, I.; Hamad-Schifferli, K.; Gehrke, L. Multicolored silver nanoparticles for multiplexed disease diagnostics: Distinguishing dengue, yellow fever, and Ebola viruses. Lab Chip 2015, 15, 1638-1641.

[183] Mosley, G. L.; Nguyen, P.; Wu, B. M.; Kamei, D. T. Development of quantitative radioactive methodologies on paper to determine important lateral-flow immunoassay parameters. Lab Chip 2016, 16, 2871-2881.

[184] OraQuick In-Home HIV Test. https://www.fda.gov/ BiologicsBloodVaccines/BloodBloodProducts/ApprovedP
roducts/PremarketApprovalsPMAs/ucm310436.htm (accessed May 7, 2018).

[185] Choi, J. R.; Tang, R. H.; Wang, S. Q.; Wan Abas, W. A. B.; Pingguan-Murphy, B.; Xu, F. Paper-based sample-to-answer molecular diagnostic platform for point-of-care diagnostics. Biosens. Bioelectron. 2015, 74, 427-439.

[186] Zhan, L.; Guo, S. Z.; Song, F. Y.; Gong, Y.; Xu, F.; Boulware, D. R.; McAlpine, M. C.; Chan, W. C. W.; Bischof, J. C. The role of nanoparticle design in determining analytical performance of lateral flow immunoassays. Nano Lett. 2017, 17, 7207-7212.

[187] Gao, Z. Q.; Ye, H. H.; Tang, D. Y.; Tao, J.; Habibi, S.; Minerick, A.; Tang, D. P.; Xia, X. H. Platinum-decorated gold nanoparticles with dual functionalities for ultrasensitive colorimetric in vitro diagnostics. Nano Lett. 2017, 17, 5572-5579.

[188] BioReady Nanoparticles for Lateral Flow. https://nanocomposix.com/collectionsbioready-nanoparticles (accessed May 7, 2018).

[189] Saha, A.; Jana, N. R. Paper-based microfluidic approach for surface-enhanced Raman spectroscopy and highly reproducible detection of proteins beyond picomolar concentration. ACS. Appl. Mater. Interfaces 2015, 7, 996-1003.

[190] Li, Y. X.; Zhang, K.; Zhao, J. J.; Ji, J.; Ji, C.; Liu, B. H. A three-dimensional silver nanoparticles decorated plasmonic paper strip for SERS detection of low-abundance molecules. Talanta 2016, 147, 493-500.

[191] Gao, X. F.; Zheng, P.; Kasani, S.; Wu, S.; Yang, F.; Lewis, S.; Nayeem, S.; Engler-Chiurazzi, E. B.; Wigginton, J. G.; Simpkins, J. W. et al. Paper-based surface-enhanced Raman scattering lateral flow strip for detection of neuronspecific enolase in blood plasma. Anal. Chem. 2017, 89, 10104-10110.

[192] Sánchez-Purrà, M.; Carré-Camps, M.; de Puig, H.; Bosch, I.; Gehrke, L.; Hamad-Schifferli, K. Surface-enhanced Raman spectroscopy-based sandwich immunoassays for multiplexed detection of Zika and Dengue viral biomarkers. ACS Infect. Dis. 2017, 3, 767-776.

[193] Zhang, Y. S.; Chang, J. B.; Alvarez, M. M.; Trujillo-de Santiago, G.; Aleman, J.; Batzaya, B.; Krishnadoss, V.; Ramanujam, A. A.; Kazemzadeh-Narbat, M.; Chen, F. et al. Hybrid microscopy: Enabling inexpensive highperformance imaging through combined physical and optical magnifications. Sci. Rep. 2016, 6, 22691.

[194] Zhang, Y. S.; Ribas, J.; Nadhman, A.; Aleman, J.; Selimović, S.; Lesher-Perez, S. C.; Wang, T.; Manoharan, V.; Shin, S. R.; Damilano, A. et al. A cost-effective fluorescence mini-microscope for biomedical applications. Lab Chip 
2015, 15, 3661-3669.

[195] Zhang, Y. S.; Trujillo-de Santiago, G.; Alvarez, M. M.; Schiff, S. J.; Boyden, E. S.; Khademhosseini, A. Expansion mini-microscopy: An enabling alternative in point-of-care diagnostics. Curr. Opin. Biomed. Eng. 2017, 1, 45-53.

[196] McLeod, E.; Ozcan, A. Microscopy without lenses. Phys. Today 2017, 70, 50-56.

[197] Ghosh, K. K.; Burns, L. D.; Cocker, E. D.; Nimmerjahn, A.; Ziv, Y.; El Gamal, A.; Schnitzer, M. J. Miniaturized integration of a fluorescence microscope. Nat. Methods 2011, 8, 871-878.

[198] Singh, N. K.; Chacko, J. V.; Sreenivasan, V. K. A.; Nag, S.; Maiti, S. Ultracompact alignment-free single molecule fluorescence device with a foldable light path. J. Biomed. Opt. 2011, 16, 025004.

[199] Cetin, A. E.; Coskun, A. F.; Galarreta, B. C.; Huang, M.; Herman, D.; Ozcan, A.; Altug, H. Handheld high-throughput plasmonic biosensor using computational on-chip imaging. Light-Sci. Appl. 2014, 3, e122.

[200] Coskun, A. F.; Cetin, A. E.; Galarreta, B. C.; Alvarez, D. A.; Altug, H.; Ozcan, A. Lensfree optofluidic plasmonic sensor for real-time and label-free monitoring of molecular binding events over a wide field-of-view. Sci. Rep. 2014, 4, 6789 .

[201] Ballard, Z. S.; Shir, D.; Bhardwaj, A.; Bazargan, S.; Sathianathan, S.; Ozcan, A. Computational sensing using low-cost and mobile plasmonic readers designed by machine learning. ACS Nano 2017, 11, 2266-2274.

[202] Taton, T. A.; Mirkin, C. A.; Letsinger, R. L. Scanometric DNA array detection with nanoparticle probes. Science 2000, 289, 1757-1760.

[203] Verigene ${ }^{\circledR}$ Warfarin Metabolism Nucleic Acid Test. Nanosphere Inc. https://www.accessdata.fda.gov/cdrh_docs/ pdf7/k070804.pdf (accessed May 7, 2018).

[204] Wei, Q. S.; Acuna, G.; Kim, S.; Vietz, C.; Tseng, D.; Chae, J.; Shir, D.; Luo, W.; Tinnefeld, P.; Ozcan, A. Plasmonics enhanced smartphone fluorescence microscopy. Sci. Rep. 2017, 7, 2124.

[205] Ozcan, A. Mobile phones democratize and cultivate next-generation imaging, diagnostics and measurement tools. Lab Chip 2014, 14, 3187-3194.

[206] Erickson, D.; O’Dell, D.; Jiang, L.; Oncescu, V.; Gumus, A.; Lee, S.; Mancuso, M.; Mehta, S. Smartphone technology can be transformative to the deployment of lab-on-chip diagnostics. Lab Chip 2014, 14, 3159-3164.

[207] Contreras-Naranjo, J. C.; Wei, Q. S.; Ozcan, A. Mobile phone-based microscopy, sensing, and diagnostics. IEEE J. Sel. Top. Quant. 2016, 22, 7100414.

[208] Breslauer, D. N.; Maamari, R. N.; Switz, N. A.; Lam, W. A.;
Fletcher, D. A. Mobile phone based clinical microscopy for global health applications. PLoS One 2009, 4, e6320.

[209] Smith, Z. J.; Chu, K. Q.; Espenson, A. R.; Rahimzadeh, M.; Gryshuk, A.; Molinaro, M.; Dwyre, D. M.; Lane, S.; Matthews, D.; Wachsmann-Hogiu, S. Cell-phone-based platform for biomedical device development and education applications. PLoS One 2011, 6, e17150.

[210] Mudanyali, O.; Dimitrov, S.; Sikora, U.; Padmanabhan, S.; Navruz, I.; Ozcan, A. Integrated rapid-diagnostic-test reader platform on a cellphone. Lab Chip 2012, 12, 2678-2686.

[211] Zhu, H. Y.; Yaglidere, O.; Su, T. W.; Tseng, D.; Ozcan, A. Cost-effective and compact wide-field fluorescent imaging on a cell-phone. Lab Chip 2011, 11, 315-322.

[212] Wei, Q. S.; Qi, H. F.; Luo, W.; Tseng, D.; Ki, S. J.; Wan, Z.; Göröcs, Z.; Bentolila, L. A.; Wu, T. T.; Sun, R. et al. Fluorescent imaging of single nanoparticles and viruses on a smart phone. ACS Nano 2013, 7, 9147-9155.

[213] Wei, Q. S.; Luo, W.; Chiang, S.; Kappel, T.; Mejia, C.; Tseng, D.; Chan, R. Y. L.; Yan, E.; Qi, H. F.; Shabbir, F. et al. Imaging and sizing of single DNA molecules on a mobile phone. ACS Nano 2014, 8, 12725-12733.

[214] Liu, Z. G.; Zhang, Y. L.; Xu, S. J.; Zhang, H.; Tan, Y. X.; Ma, C. M.; Song, R.; Jiang, L. L.; Yi, C. Q. A 3D printed smartphone optosensing platform for point-of-need food safety inspection. Anal. Chim. Acta 2017, 966, 81-89.

[215] Fu, Q. Q.; Wu, Z.; Xu, F. X.; Li, X. Q.; Yao, C. Z.; Xu, M.; Sheng, L. R.; Yu, S. T.; Tang, Y. A portable smart phone-based plasmonic nanosensor readout platform that measures transmitted light intensities of nanosubstrates using an ambient light sensor. Lab Chip 2016, 16, 1927-1933.

[216] Amirjani, A.; Fatmehsari, D. H. Colorimetric detection of ammonia using smartphones based on localized surface plasmon resonance of silver nanoparticles. Talanta 2018, 176, 242-246.

[217] Cellmic. http://www.cellmic.com/content (accessed May 7, 2018).

[218] Wei, Q. S.; Nagi, R.; Sadeghi, K.; Feng, S.; Yan, E.; Ki, S. J.; Caire, R.; Tseng, D.; Ozcan, A. Detection and spatial mapping of mercury contamination in water samples using a smart-phone. ACS Nano 2014, 8, 1121-1129.

[219] Preechaburana, P.; Gonzalez, M. C.; Suska, A.; Filippini, D. Surface plasmon resonance chemical sensing on cell phones. Angew. Chem., Int. Ed. 2012, 51, 11585-11588.

[220] Liu, Y.; Liu, Q.; Chen, S. M.; Cheng, F.; Wang, H. Q.; Peng, W. Surface plasmon resonance biosensor based on smart phone platforms. Sci. Rep. 2015, 5, 12864.

[221] Bremer, K.; Roth, B. Fibre optic surface plasmon resonance sensor system designed for smartphones. Opt. Express 2015, 23, 17179-17184. 
[222] Zhang, J. L.; Khan, I.; Zhang, Q. W.; Liu, X. H.; Dostalek, J.; Liedberg, B.; Wang, Y. Lipopolysaccharides detection on a grating-coupled surface plasmon resonance smartphone biosensor. Biosens. Bioelectron. 2018, 99, 312-317.

[223] Wang, X. H.; Chang, T. W.; Lin, G. H.; Gartia, M. R.; Liu, G. L. Self-referenced smartphone-based nanoplasmonic imaging platform for colorimetric biochemical sensing. Anal. Chem. 2017, 89, 611-615.

[224] Guner, H.; Ozgur, E.; Kokturk, G.; Celik, M.; Esen, E.; Topal, A. E.; Ayas, S.; Uludag, Y.; Elbuken, C.; Dana, A. A smartphone based surface plasmon resonance imaging (SPRi) platform for on-site biodetection. Sensor. Actuat. B: Chem. 2017, 239, 571-577.

[225] Bandodkar, A. J.; Wang, J. Non-invasive wearable electrochemical sensors: A review. Trends Biotechnol. 2014, 32, 363-371.

[226] Zhong, J. W.; Zhang, Y.; Zhong, Q. Z.; Hu, Q. Y.; Hu, B.; Wang, Z. L.; Zhou, J. Fiber-based generator for wearable electronics and mobile medication. ACS Nano 2014, 8, 6273-6280.

[227] Wang, X. W.; Gu, Y.; Xiong, Z. P.; Cui, Z.; Zhang, T. Silk-molded flexible, ultrasensitive, and highly stable electronic skin for monitoring human physiological signals. Adv. Mater. 2014, 26, 1336-1342.

[228] Xu, S.; Zhang, Y. H.; Jia, L.; Mathewson, K. E.; Jang, K. I.; Kim, J.; Fu, H. R.; Huang, X.; Chava, P.; Wang, R. H. et al. Soft microfluidic assemblies of sensors, circuits, and radios for the skin. Science 2014, 344, 70-74.

[229] Feng, D.; Zhang, H.; Xu, S. Y.; Tian, L. M.; Song, N. F. Stretchable array of metal nanodisks on a $3 \mathrm{D}$ sinusoidal wavy elastomeric substrate for frequency tunable plasmonics. Nanotechnology 2017, 28, 115703.

[230] Wei, Z. Q.; Zhou, Z. K.; Li, Q. Y.; Xue, J. C.; Di Falco, A.; Yang, Z. J.; Zhou, J. H.; Wang, X. H. Flexible nanowire cluster as a wearable colorimetric humidity sensor. Small 2017, 13, 1700109.

[231] Yan, H. G.; Low, T.; Zhu, W. J.; Wu, Y. Q.; Freitag, M.; Li, X. S.; Guinea, F.; Avouris, P.; Xia, F. N. Damping pathways of mid-infrared plasmons in graphene nanostructures. Nat. Photonics 2013, 7, 394-399.

[232] Yan, H. G.; Li, X. S.; Chandra, B.; Tulevski, G.; Wu, Y. Q.; Freitag, M.; Zhu, W. J.; Avouris, P.; Xia, F. N. Tunable infrared plasmonic devices using graphene/insulator stacks. Nat. Nanotechnol. 2012, 7, 330-334.

[233] Leem, J.; Wang, M. C.; Kang, P.; Nam, S. Mechanically self-assembled, three-dimensional graphene-gold hybrid nanostructures for advanced nanoplasmonic sensors. Nano Lett. 2015, 15, 7684-7690.

[234] Hinman, S. S.; McKeating, K. S.; Cheng, Q. Plasmonic sensing with 3D printed optics. Anal. Chem. 2017, 89, 12626-12630.

[235] Pandey, S.; Gupta, B.; Nahata, A. Terahertz plasmonic waveguides created via 3D printing. Opt. Express 2013, $21,24422-24430$. 